\title{
On Algebraic Supergroups, Coadjoint Orbits and their Deformations
}

\author{
R. Fioresi ${ }^{1}$ \\ Dipartimento di Matematica, Università di Bologna \\ Piazza di Porta S. Donato, 5. \\ 40126 Bologna. Italy. \\ e-mail: fioresi@dm.UniBo.it \\ and \\ M. A. Lledó \\ INFN, Sezione di Torino, Italy and \\ Dipartimento di Fisica, Politecnico di Torino, \\ Corso Duca degli Abruzzi 24, I-10129 Torino, Italy. \\ e-mail: lledo@athena.polito.it
}

\begin{abstract}
In this paper we study algebraic supergroups and their coadjoint orbits as affine algebraic supervarieties. We find an algebraic deformation quantization of them that can be related to the fuzzy spaces of non commutative geometry.
\end{abstract}

\footnotetext{
${ }^{1}$ Investigation supported by the University of Bologna, funds for selected research topics.
} 


\section{Introduction}

The use of "odd variables" in physics is very old and quite natural. It is unavoidable in the description of theories involving particles like the electron. Physical theories are given by functionals on a certain kind of "fields" or sections of a bundle over the manifold of spacetime $\mathcal{M}$. The bundle has fibers which are super vector spaces ( $\mathbb{Z}_{2}$-graded vector spaces), and that have a commutative superalgebra structure. In an open set $U \subset \mathcal{M}$ of spacetime the set of fields can be written as

$$
\left\{\phi_{i}(x), \psi_{\alpha}(x)\right\}, \quad i=1, \ldots p, \alpha=1, \ldots q, \quad x \in U
$$

with the superalgebra structure given by the relations

$$
\begin{gathered}
\phi_{i}(x) \phi_{j}(x)-\phi_{j}(x) \phi_{i}(x)=0, \quad \phi_{i}(x) \psi_{\alpha}(x)-\psi_{\alpha}(x) \phi_{i}(x)=0, \\
\psi_{\alpha}(x) \psi_{\beta}(x)+\psi_{\beta}(x) \psi_{\alpha}(x)=0 .
\end{gathered}
$$

Free fields (i.e. fields satisfying the free equations of motion) define unitary representations of the Poincaré group. It was soon realized that in order to have a consistent description, the half integer spin representations had to be described by odd valued fields while the integer spin representations were described by even valued fields. This is known as the spin-statistics theorem.

Once the necessity of odd or Grassmann coordinates was established on physical grounds it would have been natural to consider superspaces or supermanifolds. Nevertheless, there was still an important conceptual step to be taken, and the input came also from physics. To have a supermanifold, all the coordinates, even and odd, must be put on equal grounds. This means that changes of coordinates mix together even and odd quantities in a consistent way. From the physics point of view, this would mean that fields with different spin and statistics could be mixed by certain transformations. But all the groups of symmetries considered in physics until then would preserve the even and odd subspaces, not allowing such a mixing. They were all even transformations or super vector space morphisms, since they would not change the degree of the vector on which they act. The first example of super transformations, that is, transformations that mix the odd and even subspaces irreducibly, appeared in the seminal paper by Gol'fand and Likhtman [1]. (Other examples of Lie superalgebras had appeared in the mathematical literature before, but not related to trasformation groups). In that paper, an extension of the Poincaré algebra by means of a set of odd 
translations whose anticommutator is proportional to an ordinary translation was constructed. To comply with the spin-statistics theorem, the generators of the odd translations where in a spin 1/2 representation of the Poincaré group. This was the first example in physics of a Lie superalgebra.

Natural as it seems from this historical perspective, the introduction of Lie superalgebras (and Lie supergroups) has been revolutionary in physics. The assumption that supersymmetry (that is, symmetry under a supergroup of transformations) is realized in nature has profound implications, since it restricts significantly the number of theories that can be considered. On the other hand, supersymmetric field theories have many advantages in the quest for a unified theory of all interactions. Accordingly, there is a huge number of works dealing with supersymmetric field theories. Some pioneering works are the one by Wess and Zumino [2] on the chiral multiplet, by Salam and Strathdee [3] and Ferrara, Wess and Zumino [4 on superspace, by Wess and Zumino [5] on supersymmetric electrodynamics, by Ferrara and Zumino [6] and Salam and Strathdee [7] on supersymmetric Yang-Mills theories [6], and by Ferrara, Freedman and van Nieuwenhuizen [8] and Deser and Zumino [9] on supergravity. Further information and references can be found in the collected reprint volume [10].

Since those first days the concept of supermanifold in mathematics has evolved to a very precise formulation. Starting with the superanalysis of Berezin [11, and together with other works by Kostant [12, Leites [13, Manin [14], Bernstein, Deligne and Morgan [15] to mention some of the most representative. Supermanifolds are seen roughly as ordinary manifolds together with a sheaf of superalgebras. The odd coordinates appear in the stalks of the sheaf. This approach allows considerable freedom. Following Manin [14, one can define different kinds of superspaces, supermanifolds or algebraic supervarieties by choosing a base space with the appropriate topology. The sheaves considered are superalgebra valued, so that one can generalize the concepts of complex and algebraic geometry to this new setting.

In this paper we will deal strictly with algebraic supervarieties, but the concept of supermanifold has been treated extensively in the literature mentioned above.

It is interesting to note that there is an alternative definition of algebraic variety in terms of its functor of points. Essentially, an algebraic variety can be defined as a certain functor from some category of commutative algebras 
to the category of sets. It is then very natural to substitute the category of algebras by an appropriate category of commutative superalgebras and to call this a supervariety. The same can be done for supergroups, super Lie algebras, coadjoint orbits of supergroups and other "super" objects. The elegance of this approach cannot hide the many non trivial steps involved in the generalization. As an example, it should be enough to remember the profound differences between the classifications of semisimple Lie algebras and semisimple Lie superalgebras [11, 16, 17].

The purpose of this paper is to study the coadjoint orbits of certain supergroups, to establish their structure as algebraic supermanifolds and to obtain a quantum deformation of the superalgebras that represent them.

Recently there has been a growing interest in the physics literature on non commutative spaces (also called "fuzzy spaces"). The idea that spacetime may have non commuting coordinates which then cannot be determined simultaneously has been proposed at different times for diverse motives [20, 21, 22, 23]. In particular, these spaces have been considered as possible compactification manifolds of string theory [23].

In general, we can say that a "fuzzy" space is an algebra of operators on a Hilbert space obtained by some quantization procedure. This means that it is possible to define a classical limit for such algebra, which will be a commutative algebra with a Poisson structure, that is, a phase space. As it is well known, the coadjoint orbits of Lie groups are symplectic manifolds and hamiltonian spaces with the Kirillov Poisson structure of the corresponding Lie group. There is a wide literature on the quantization of coadjoint orbits, most of the works based on the Kirillov-Kostant orbit principle which associates to every orbit (under some conditions) a unitary representation of the group. Let us consider as an example the sphere $S^{2}$, a regular coadjoint orbit of the group $\mathrm{SU}(2)$. In physical terms, the quantization of $S^{2}$ is the quantization of the spin; it is perhaps the most classical example of quantization, other than flat space $\mathbb{R}^{2 n}$. In the approach of geometric quantization (see Ref. [18 for a review) the sphere must have half integer radius $j$ and to this orbit it corresponds the unitary representation of $\mathrm{SU}(2)$ of spin $j$ (which is finite dimensional). Let us take the classical algebra of observables to be the polynomials on the algebraic variety $S^{2}$. Given that the Hilbert space of the quantum system is finite dimensional, the algebra of observables is also finite dimensional. It is in fact of dimension $(2 j+1)^{2}$ and isomorphic to the algebra of $(2 j+1) \times(2 j+1)$-matrices [19, 20]. After a rescaling of the coordinates, we can take the limit $j \rightarrow \infty$ maintaining the radius of the sphere constant. 
In this way the algebra becomes infinite dimensional and all polynomials are quantized. This procedure is most appropriately described by Madore [20, being the algebras with finite $j$ approximations to the non commutative or fuzzy sphere.

The deformation quantization approach [24] is inspired in the correspondence principle: to each classical observable there must correspond a quantum observable (operator on a Hilbert space). This quantization map will induce a non commutative, associative star product on the algebra of classical observables which will be expressed as a power series in $\hbar$, having as a zero order term the ordinary, commutative product and as first order term the Poisson bracket. One can ask the reverse question, and explore the possible deformations of the commutative product independently of any quantization map or Hilbert space. It is somehow a semiclassical approach. Very often, the star product is only a formal star product, in the sense that the series in $\hbar$ does not converge. It however has the advantage that many calculations and questions may be posed in a simpler manner (see [32] for the string theory application of the star product).

The deformation quantization of the sphere and other coadjoint orbits has been treated in the literature [25, 26, 27, 28, 29]. The immediate question is if deformation theory can give some information on representation theory and the Kirillov-Kostant orbit principle 30, 31.

In Ref. 28] a family of algebraic star products is defined on regular orbits of semisimple groups. The star product algebra is isomorphic to the quotient of the enveloping algebra by some ideal. It is in fact possible to select this ideal in such a way that it is in the kernel of some unitary representation. The image of this algebra by the representation map will give a finite dimensional algebra. In this way we can obtain the fuzzy sphere and other fuzzy coadjoint orbits from the star product. The star product is then seen as a structure underlying and unifying all the finite dimensional fuzzy algebras. The classical limit and the correspondence principle are seen naturally in this approach.

In this paper we extend the approach of Ref. [28] to some coadjoint orbits of some semisimple supergroups. The extension to the super category is not straightforward and this is reflected in the fact that we have to restrict ourselves to the supergroups $\mathrm{SL}_{m \mid n}$ and $\mathrm{OSp}_{m \mid n}$, and orbits of elements with distinct eigenvalues (see Theorem 4.1) to obtain the extension. This is by no means an affirmation that the procedure could not be in principle be applied to more general supergroups and orbits, but a consequence of the technical 
difficulties involved.

Other treatments of the "fuzzy supershere" can be found in Refs. 35, 34, 33. A comparison with these approaches as well as a relation of our star product with representation theory of supergroups will be discussed elsewhere.

The paper is organized as follows: Section 2 is dedicated to the definition of algebraic supervariety and its functor of points. The definitions and results that we mention are somehow scattered in the literature and we want to give a comprehensive account here [36, 20. Section 3 is dedicated to algebraic supergroups and their associated Lie superalgebras in terms of their functors. The correspondence between the algebraic Lie supergroup and its Lie superalgebra is treated in detail. Most of the definitions and theorems extend easily from the classical case, but we are not aware of any reference where this account has been done explicitly for the algebraic case (instead, the differential case is better known). We show proofs when we think it can help to read the paper. We also illustrate the abstract definitions with the examples of $\mathrm{GL}_{m \mid n}$ and $\mathrm{SL}_{m \mid n}$. In Section 4 we retrieve the coadjoint orbits of the supergroups mentioned above as certain representable functors, and then as affine algebraic supervarieties. Finally, in Section 5 we present a deformation of the superalgebra that represents the functor associated to the coadjoint orbit.

\section{$2 \quad$ Algebraic supervarieties and superschemes}

In this section we want to give a definition of algebraic supervarieties and superschemes. Our description is self-contained and very much inspired in the approach of Refs. [14, 15]. We assume some knowledge of basic algebraic geometry and of super vector calculus. We are especially interested in the description of a supervariety in terms of its functor of points.

Let $k$ be a commutative ring. All algebras and superalgebras will be intended to be over $k$ and are assumed to be commutative unless otherwise stated. If $A$ is a superalgebra we will denote by $A_{0}$ the even part and by $A_{1}$ the odd part, so $A=A_{0}+A_{1}$. Let $\mathcal{I}_{A}^{\text {odd }}$ be the ideal in $A$ generated by the odd part. The quotient $A^{\circ}=A / \mathcal{I}_{A}^{\text {odd }}$ is an ordinary algebra. Notice that $A$ is both, an $A_{0}$-module and an $A^{\circ}$-module.

If $\mathcal{O}_{X}$ is a sheaf of superalgebras over a topological space $X$, then $\mathcal{O}_{X, 0}$ 
and $\mathcal{O}_{X}^{\circ}$ are sheaves of algebras with

$$
\mathcal{O}_{X, 0}(U)=\mathcal{O}_{X}(U)_{0}, \quad \mathcal{O}_{X}^{\circ}(U)=\mathcal{O}_{X}(U)^{\circ} \quad U \subset_{\text {open }} X .
$$

We denote by $\left(\operatorname{alg}_{\mathrm{rf}}\right)$ the category of commutative $k$-algebras which are reduced $^{2}$ and finitely generated (often called affine algebras), and by ( $\operatorname{salg}_{\mathrm{rf}}$ ) the category of commutative $k$-superalgebras finitely generated, and such that, modulo the ideal generated by the odd elements, they are reduced.

We denote also by (alg) and (salg) the categories of commutative $k$ algebras and commutative $k$-superalgebras respectively.

\section{$2.1 \quad$ Ringed superspaces}

Definition 2.1 A superspace $\left(X, \mathcal{O}_{X}\right)$ is a topological space $X$ together with a sheaf of superalgebras $\mathcal{O}_{X}$, such that:

a. $\left(X, \mathcal{O}_{X, 0}\right)$ is a noetherian scheme,

b. $\mathcal{O}_{X}$ is a coherent sheaf of $\mathcal{O}_{X, 0}$-modules.

Definition 2.2 A morphism of superspaces $\left(X, \mathcal{O}_{X}\right)$ and $\left(Y, \mathcal{O}_{Y}\right)$ is given by a pair $(f, \psi)$ where $f: X \rightarrow Y$ is a continuous map, $\psi: \mathcal{O}_{Y} \rightarrow f_{*} \mathcal{O}_{X}$ is a map of sheaves of superalgebras on $Y$ and $\left(f,\left.\psi\right|_{\mathcal{O}_{Y}}\right)$ is a morphism of schemes.

$f_{*} \mathcal{O}_{X}$ denotes the push-forward of the sheaf $\mathcal{O}_{X}$ under $f$.

\section{Example 2.1}

Let $A$ be an object of (salg). We consider the topological space $X_{A}:=$ $\operatorname{Spec}\left(A_{0}\right)=\operatorname{Spec}\left(A^{\circ}\right)$ (they are isomorphic since the algebras differ only by nilpotent elements) with the Zariski topology.

On $X_{A}$ we have the structural sheaf, $\mathcal{O}_{A_{0}}$. The stalk of the sheaf at the prime $\mathbf{p} \in \operatorname{Spec}\left(A_{0}\right)$ is the localization of $A_{0}$ at $\mathbf{p}$. As for any superalgebra, $A$ is a module over $A_{0}$, so we have indeed a sheaf of $\mathcal{O}_{A_{0}}$-modules over $X_{A}$ with stalk the localization of the $A_{0}$-module $A$ over each prime $\mathbf{p} \in \operatorname{Spec}\left(A_{0}\right)$. It is a sheaf of superalgebras that we will denote by $\tilde{A} .\left(X_{A}, \tilde{A}\right)$ is a superspace. (For more details on the construction of the sheaf $\tilde{M}$, for a generic $A_{0}$-module $M$, see [37] II $\S 5)$.

\footnotetext{
${ }^{2} \mathrm{~A}$ reduced algebra is an algebra that has no nilpotent elements.
} 
Definition 2.3 An affine algebraic supervariety is a superspace isomorphic to $\left(X_{A}, \tilde{A}\right)$ for some superalgebra $A$ in $\left(\operatorname{salg}_{\mathrm{rf}}\right)$.

$A$ is often called the coordinate superalgebra of the supervariety. The affine algebraic supervarieties form a category denoted by $\left(\right.$ svar $\left._{\text {aff }}\right)$. Given an affine algebraic supervariety $\left(V, \mathcal{O}_{V}\right)$ (or just $V$ for short) we have an ordinary affine algebraic variety associated to it, $\left(V, \mathcal{O}_{V}^{\circ}\right)$. It is called the reduced variety of $V$ and denoted also by $V^{\circ}$.

It is straightforward to generalize the construction to an arbitrary commutative superalgebra $A$. We then have the following

Definition 2.4 An affine superscheme is a superspace isomorphic to $\left(X_{A}, \tilde{A}\right)$ for some superalgebra $A$ in (salg).

The affine superschemes over $k$ form a category denoted by (sschemes aff $_{\text {) }}$. As in the classical case, general supervarieties and superschemes are defined as superspaces that are locally affine supervarieties and affine superschemes.

To any superscheme $\left(S, \mathcal{O}_{S}\right)$ one can associate an ordinary scheme $\left(S, \mathcal{O}_{S}^{\circ}\right)$, called the reduced scheme.

Example 2.2 Supervariety over the sphere $S^{2}$.

(See Ref.[1], page 8). This is an example of a supervariety explicitly given by the superalgebra representing the functor. We will see how the sheaf is constructed in the closed points (maximal ideals) of the topological space. Consider the free commutative superalgebra generated by three even variables $x_{1}, x_{2}, x_{3}$ and three odd variables $\xi_{1}, \xi_{2}, \xi_{3}, k\left[x_{1}, x_{2}, x_{3}, \xi_{1}, \xi_{2}, \xi_{3}\right]$, and the ideal

$$
\mathcal{I}=\left(x_{1}^{2}+x_{2}^{2}+x_{3}^{2}-1, x_{1} \cdot \xi_{1}+x_{2} \cdot \xi_{2}+x_{3} \cdot \xi_{3}\right) .
$$

The superalgebra $k[V]=k\left[x_{1}, x_{2}, x_{3}, \xi_{1}, \xi_{2}, \xi_{3}\right] / \mathcal{I}$ represents a supervariety whose reduced variety $V^{\circ}$ is the sphere $S^{2}$. At each maximal ideal in $k[V]_{0}$,

$$
\mathbf{m}=\left(x_{i}-a_{i}, \xi_{i} \xi_{j}\right) \quad \text { with } i, j=1,2,3, \quad a_{i} \in k \text { and } a_{1}^{2}+a_{2}^{2}+a_{3}^{2}=1,
$$

the local ring of $k[V]_{0}$ is the ring of fractions

$$
\left(k[V]_{0}\right)_{\mathbf{m}}=\left\{\frac{f}{g} / f, g \in k[V]_{0}, g \notin \mathbf{m}\right\} .
$$


The stalk of the structural sheaf at $\mathbf{m}$ is the localization of $k[V]$ as a $k[V]_{0^{-}}$ module, that is

$$
k[V]_{\mathbf{m}}=\left\{\frac{m}{g} / m \in k[V], g \in k[V]_{0}, g \notin \mathbf{m}\right\} .
$$

Notice that if $a_{1} \neq 0$ (not all $a_{i}$ are zero simultaneously), then $x_{1}$ is invertible in the localization and we have

$$
\xi_{1}=\frac{1}{x_{1}}\left(x_{2} \xi_{2}+x_{3} \xi_{3}\right)
$$

so $\left\{\xi_{2}, \xi_{3}\right\}$ generate $k[V]_{\mathbf{m}}$ as an $\mathcal{O}_{k[V]_{0}}$-module.

\subsection{The functor of points}

We recall first the definition of the functor of points in the classical (non super) case.

Let $X$ be an affine variety. The representable functor $h_{X}:\left(\operatorname{var}_{\mathrm{aff}}\right)^{\mathrm{opp}} \rightarrow$ (sets) ${ }^{3}$ from the category of affine varieties to the category of sets

$$
h_{X}(Y)=\operatorname{hom}_{\left(\operatorname{var}_{\text {aff }}\right)}(Y, X)
$$

is the functor of points of $X$. An element of $\operatorname{hom}_{\left(\operatorname{var}_{\mathrm{aff}}\right)}(Y, X)$ is an $Y$-point of $X$. Given the equivalence of categories

$$
\begin{aligned}
F:\left(\operatorname{alg}_{\mathrm{rf}}\right) & \longrightarrow\left(\operatorname{var}_{\mathrm{aff}}\right) \\
R & \longrightarrow \operatorname{Spec}(R),
\end{aligned}
$$

we can equivalently define the functor of points of an affine variety $X=$ $\operatorname{Spec}(R)$ as the representable functor

$$
h_{R}(T)=\operatorname{hom}_{\left(\operatorname{alg}_{\mathrm{rf}}\right)}(R, T), \quad T \in\left(\operatorname{alg}_{\mathrm{rf}}\right) .
$$

This leads to an alternative definition of affine variety as a representable functor between the categories $\left(\operatorname{alg}_{\mathrm{rf}}\right)$ and (sets).

These definitions and observations can be extended immediately to the super case.

\footnotetext{
${ }^{3}$ By the label opp we denote the category with the direction of morphisms inverted. We could equally speak about contravariant functors.
} 
Definition 2.5 The functor of points of a supervariety $X$ is a representable functor

$$
h_{X}:\left(\text { svar }_{\mathrm{aff}}\right)^{\mathrm{opp}} \rightarrow(\text { sets }) .
$$

\section{Observation 2.1}

The construction of Example 2.1 defines an equivalence of categories between $\left(\right.$ svar $\left._{\text {aff }}\right)$ and $\left(\operatorname{salg}_{\mathrm{rf}}\right)$ and between ( sschemes $\left._{\text {aff }}\right)$ and (salg). So the functor of points can be equivalently be given by a representable functor $h_{A}:\left(\operatorname{salg}_{\mathrm{rf}}\right) \rightarrow$ (sets). For example, as we will see in the next section, algebraic supergroups can easily be defined as certain representable functors.

In the general case, for a general superscheme $X$, its functor of points can be defined either as a representable functor from (sschemes) ${ }^{\text {opp }} \rightarrow$ (sets) $^{\circ}$ or as the functor

$$
h_{X}:(\mathrm{salg})^{\mathrm{opp}} \longrightarrow(\mathrm{sets}), \quad h_{X}(A)=\operatorname{hom}_{(\text {sschemes })}\left(X_{A}, X\right)
$$

where $X_{A}$ is the affine superscheme associated to the superalgebra $A$ (see Example 2.11). In fact, as it happens in the non super case (see Ref. [4] pg 253), the functor of points of a superscheme is determined by looking at its restriction to affine superschemes. Since we are mainly interested in affine supervarieties and affine superschemes we will not pursue further this subject.

\section{Algebraic supergroups and their Lie su- peralgebras}

In this section we generalize the basic notions of algebraic groups to the super case.

\subsection{Supergroups and supergroup functors}

Definition 3.1 An affine algebraic supergroup is a representable, group valued functor

$$
G:\left(\operatorname{salg}_{\mathrm{rf}}\right) \rightarrow(\text { sets })
$$


The superalgebra $k[G]$ representing a supergroup has the additional structure of a commutative super Hopf algebra. The coproduct

$$
\begin{aligned}
\Delta: k[G] & \longrightarrow k[G] \otimes k[G] \\
f & \longrightarrow \Delta f
\end{aligned}
$$

is such that for any affine superalgebra $A$ and two morphisms ( $A$-points) $x, y \in G(A)=\operatorname{hom}_{k-\operatorname{superalg}}(k[G], A)$ the following relation holds:

$$
m_{A}(x \otimes y(\Delta f))=x \cdot y(f) \quad \forall f \in k[G]
$$

where $m_{A}$ denotes the multiplication in $A$ and "." denotes the multiplication in the group $G(A)$. The tensor product is understood in the tensor category of super vector spaces [15]. The counit is given by

$$
\begin{aligned}
& \mathcal{E}: k[G] \longrightarrow k \\
& f \longrightarrow \quad e(f)
\end{aligned}
$$

where $e$ is the identity in $G(k)$. The antipode is defined as

$$
\begin{aligned}
S: & k[G] \\
f & \longrightarrow[G] \\
& S(f)
\end{aligned}
$$

with $x(f)=x^{-1}(S(f)) \quad \forall x \in G(A)$, and where $x^{-1}$ denotes the group inverse of $x$ in $G(A)$.

Example 3.1 The supergroups $\mathrm{GL}_{m \mid n}$ and $\mathrm{SL}_{m \mid n}$.

Let $A$ be a commutative superalgebra. We denote by $A^{m \mid n}$ the free module over $A$ generated by $m$ even generators and $n$ odd generators. The endomorphisms of this super vector space (linear maps that preserve the grading) are given by matrices (we use the conventions of Ref. [15])

$$
\left(\begin{array}{cc}
p_{m \times m} & q_{m \times n} \\
r_{n \times m} & s_{n \times n}
\end{array}\right)
$$

where $p$ and $s$ have even entries and $q$ and $r$ have odd entries in $A$. We denote the set of these matrices as $\mathfrak{g l}_{m \mid n}(A)$. We can define a functor

$$
\begin{aligned}
\mathfrak{g l}_{m \mid n}:\left(\operatorname{salg}_{\mathrm{rf}}\right) & \longrightarrow(\text { sets }) \\
A & \longrightarrow \mathfrak{g l}_{m \mid n}(A) .
\end{aligned}
$$


$\mathfrak{g l}_{m \mid n}$ is a representable functor. It is represented by the superalgebra

$$
k\left[\mathfrak{g l}_{m \mid n}\right]:=k\left[x_{i j}, y_{\alpha \beta}, \xi_{i \beta}, \gamma_{\alpha j}\right], \quad i, j=1, \ldots m, \quad \alpha, \beta=1, \ldots n
$$

where $x$ and $y$ are even generators and $\xi$ and $\gamma$ are odd generators. In fact, writing the generators in matrix form,

$$
\left(\begin{array}{ll}
\left\{x_{i j}\right\} & \left\{\xi_{i \beta}\right\} \\
\left\{\gamma_{\alpha j}\right\} & \left\{y_{\alpha \beta}\right\}
\end{array}\right)
$$

any matrix as in (11) assigns to a generator of $k\left[\mathfrak{g l}_{m \mid n}\right]$ an element of $A$, and the assignment has the right parity. Hence, it defines a superalgebra morphism $k\left[\mathfrak{g l}_{m \mid n}\right] \rightarrow A$.

$\mathrm{GL}_{m \mid n}(A)$ is defined as the set of all morphisms $g: A^{m \mid n} \rightarrow A^{m \mid n}$ which are invertible. In terms of the matrix (11), this means that the Berezinian [1] or superdeterminant

$$
\operatorname{Ber}(g)=\operatorname{det}\left(p-q s^{-1} r\right) \operatorname{det}\left(s^{-1}\right)
$$

is invertible in $A$. A necessary and sufficient condition for $g$ to be invertible is that $p$ and $s$ are invertible. The group valued functor

$$
\begin{aligned}
\mathrm{GL}_{m \mid n}:\left(\operatorname{salg}_{\mathrm{rf}}\right) & \longrightarrow(\text { sets }) \\
A & \longrightarrow \mathrm{GL}_{m \mid n}(A) .
\end{aligned}
$$

is an affine supergroup represented by the algebra [40]

$$
\begin{gathered}
k\left[\mathrm{GL}_{m \mid n}\right]:=k\left[x_{i j}, y_{\alpha \beta}, \xi_{i \beta}, \gamma_{\alpha j}, z, w\right] /((w \operatorname{det}(x)-1, z \operatorname{det}(y)-1), \\
i, j=1, \ldots m, \quad \alpha, \beta=1, \ldots n .
\end{gathered}
$$

Requiring that the berezinian is equal to 1 gives the supergroup $\mathrm{SL}_{m \mid n}$, represented by

$$
k\left[\mathrm{GL}_{m \mid n}\right] /\left(\operatorname{det}\left(x-\xi y^{-1} \gamma\right) z-1\right)
$$

where $y^{-1}$ is the matrix of indeterminates, inverse of the matrix $y$, whose determinant is invertible and has inverse $z$.

In Ref. 40] the Hopf superalgebra structure of this affine supergroup was explicitly computed.

In the classical case, the concept of group functor is a generalization of the concept of algebraic group. This is treated extensively in [42] II, §1. It will be useful to introduce this notion for the super case, which can be done easily with suitable changes. 
Definition 3.2 Let $G$ be a functor from (salg) to (sets). We say that $G$ is a supergroup functor if:

1. There exists a natural transformation called the composition law

$$
m: G \times G \longrightarrow G
$$

satisfying the associativity condition: $m \cdot(m \times \mathbb{1})=m \cdot(\mathbb{1 1} \times m)$.

2. There exists a natural transformation, the unit section, $u: e_{k} \longrightarrow G$, where $e_{k}:(\mathrm{salg}) \longrightarrow$ (sets), $e_{k}(A)=1_{A}$, satisfying the commutative diagram:

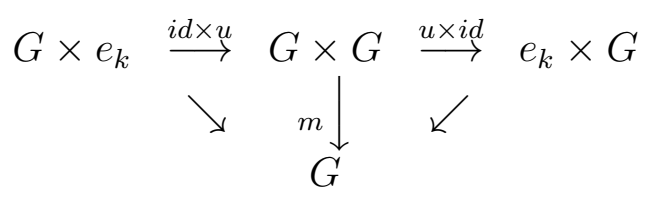

3. There exists a natural transformation $\sigma: G \longrightarrow G$ satisfying:

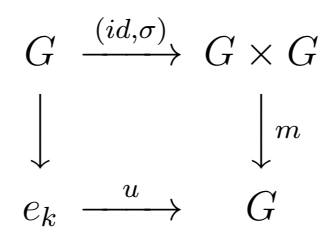

A morphism of supergroup functors is defined as a natural transformation preserving the composition law.

Because of their representability property, affine algebraic supergroups are supergroup functor.

\subsection{Lie superalgebras}

Let $\mathcal{O}_{k}:\left(\operatorname{salg}_{\mathrm{rf}}\right) \longrightarrow$ (sets) be the functor represented by $k[x] . k[x]$ corresponds in fact to an ordinary algebraic variety, $\operatorname{Spec}(k[x])$, the affine line. For a superalgebra $A$ we have that $\mathcal{O}_{k}(A)=A_{0}$.

Definition 3.3 A Lie superalgebra is a representable, group valued functor

$$
\mathfrak{g}:\left(\operatorname{salg}_{\mathrm{rf}}\right) \longrightarrow(\text { sets })
$$

with the following properties:

1. $\mathfrak{g}$ has the structure of $\mathcal{O}_{k}$-module, that is, there is a natural transformation $\mathcal{O}_{k} \times \mathfrak{g} \longrightarrow \mathfrak{g}$. For each superalgebra $A$ we have an $A_{0}$-module structure on $\mathfrak{g}(A)$. 
2. There is a natural transformation [, ]: $\mathfrak{g} \times \mathfrak{g} \longrightarrow \mathfrak{g}$ which is $\mathcal{O}_{k}$-linear and that satisfies commutative diagrams corresponding to the antisymmetric property and the Jacobi identity. For each superalgebra A, [, ] defines a Lie algebra structure on $\mathfrak{g}(A)$, hence the functor $\mathfrak{g}$ is Lie algebra valued.

For any algebraic supergroup there is a Lie superalgebra which is naturally associated. It is our purpose to construct it explicitly. Again, the construction is a generalization (not completely straightforward) of what happens in the non super case. Our treatment is very similar to the one in Ref. [15] II, 4, no 1 .

Let $A$ be a commutative superalgebra and let $A(\epsilon)={ }_{\text {def }} A[\epsilon] /\left(\epsilon^{2}\right)$ be the algebra of dual numbers ( $\epsilon$ here is taken as an even indeterminate). We have that $A[\epsilon]=A \oplus \epsilon A$ and there are two homorphisms: $i: A \rightarrow A(\epsilon)$ defined by $i(1)=1$ and $p: A(\epsilon) \rightarrow A$ defined by $p(1)=1, p(\epsilon)=0$.

Definition 3.4 Let $G$ be a supergroup functor. Consider the homomorphism $G(p): G(A(\epsilon)) \longrightarrow G(A)$. For each $G$ there is a supergroup functor, $\operatorname{Lie}(G)$, defined as

$$
\operatorname{Lie}(G)(A)=_{\text {def }} \operatorname{ker}(G(p)) .
$$

We will see that when $G$ is an affine algebraic supergroup (so it is a representable group functor) $\operatorname{Lie}(G)$ is indeed a Lie superalgebra.

It is instructive to see first an example.

Example 3.2 Lie $\left(\mathrm{GL}_{m \mid n}\right)$, Lie $\left(\mathrm{SL}_{m \mid n}\right)$

We want to determine the functor $\operatorname{Lie}\left(\mathrm{GL}_{m \mid n}\right)$. Consider the map:

$$
\begin{aligned}
& \mathrm{GL}_{m \mid n}(p): \quad \mathrm{GL}_{m \mid n}(A(\epsilon)) \quad \longrightarrow \mathrm{GL}_{m \mid n}(A) \\
& \left(\begin{array}{ll}
p+\epsilon p^{\prime} & q+\epsilon q^{\prime} \\
r+\epsilon r^{\prime} & s+\epsilon s^{\prime}
\end{array}\right) \mapsto \quad\left(\begin{array}{ll}
p & q \\
r & s
\end{array}\right)
\end{aligned}
$$

with $p, p^{\prime}, s, s^{\prime}$ having entries in $A_{0}$ and $q, q^{\prime}, r, r^{\prime}$ having entries in $A_{1} . p$ and $s$ are invertible matrices. One can see immediately that

$$
\operatorname{Lie}\left(\mathrm{GL}_{m \mid n}\right)(A)=\operatorname{ker}\left(\mathrm{GL}_{m \mid n}(p)\right)=\left\{\left(\begin{array}{cc}
1+\epsilon p^{\prime} & \epsilon q^{\prime} \\
\epsilon r^{\prime} & 1+\epsilon s^{\prime}
\end{array}\right)\right\}
$$

The functor Lie $\left(\mathrm{GL}_{m \mid n}\right)$ is clearly group valued and can be identified with the (additive) group functor $\mathfrak{g l}_{m \mid n}$ (see Example 3.1). 
For $\operatorname{Lie}\left(\mathrm{SL}_{m \mid n}\right)$ one gets the extra condition Ber $=\operatorname{det}\left(1+\epsilon p^{\prime}\right) \operatorname{det}(1-$ $\left.\epsilon s^{\prime}\right)=1$, which implies the zero supertrace condition, that is, $\operatorname{tr}\left(p^{\prime}\right)-\operatorname{tr}\left(s^{\prime}\right)=$ 0 . The functor Lie $\left(\mathrm{SL}_{m \mid n}\right)$ is then identified with the (additive) group functor

$$
\mathfrak{s l}_{m \mid n}(A)=\left\{x \in \mathfrak{g l}_{m \mid n}(A) / \operatorname{str}(x)=0\right\} .
$$

The functors $\mathfrak{g l}_{m \mid n}$ and $\mathfrak{s l}_{m \mid n}$ are representable and Lie algebra valued. We have already seen that $\mathfrak{g l}_{m \mid n}$ is representable (see Example 3.1) $\cdot \mathfrak{s l}_{m \mid n}$ is represented by the superalgebra:

$$
k\left[\mathfrak{s l}_{m \mid n}\right]=k\left[\mathrm{M}_{m \mid n}\right] / \operatorname{str}(m)
$$

where $m$ is the matrix of indeterminates generating the algebra $k\left[\mathfrak{g l}_{m \mid n}\right]$.

We want to show that when $G$ is an affine algebraic group then $\operatorname{Lie}(G)$ is a superalgebra. We will show first that the functor satisfies both properties in Definition 3.3. Then we will show that it is representable. We start by seeing that $\operatorname{Lie}(G)$ has a structure of $\mathcal{O}_{k}$-module.

Let $u_{a}: A(\epsilon) \longrightarrow A(\epsilon)$ be the endomorphism, $u_{a}(1)=1, u_{a}(\epsilon)=a \epsilon$, for $a \in A_{0}$. $\operatorname{Lie}(G)$ admits a $\mathcal{O}_{k}$-module structure, i.e. there is a natural transformation

$$
\mathcal{O}_{k} \times \operatorname{Lie}(G) \longrightarrow \operatorname{Lie}(G)
$$

such that for any superalgebra $A, a \in \mathcal{O}_{k}(A), x \in \operatorname{Lie}(G)(A)$,

$$
(a, x) \mapsto a x=\operatorname{Lie}(G)\left(u_{a}\right) x .
$$

Notice that for subgroups of $\mathrm{GL}_{m \mid n}(A)$, ax corresponds to the multiplication of the matrix $x$ by the even scalar $a$.

Let us consider the group of linear automorphisms of $\operatorname{Lie}(G)(A)$. Because of the natural $\mathcal{O}_{k}$-module structure of $\operatorname{Lie}(G)$ we have a group functor

$$
\mathrm{GL}(\operatorname{Lie}(G)):\left(\operatorname{salg}_{\mathrm{rf}}\right) \longrightarrow(\operatorname{sets}) \text {. }
$$

Alternatively one can also denote $\operatorname{GL}(\operatorname{Lie}(G))=\operatorname{Aut}(\operatorname{Lie}(G))$.

We now want to introduce the Lie algebra structure (property 2 in Definition 3.3). We will do it through the adjoint actions, seen as natural transformations.

Definition 3.5 Let $G$ be a supergroup functor. The adjoint action of $G$ on $\operatorname{Lie}(G)$ is defined as the natural transformation

$$
G \stackrel{\text { Ad }}{\longrightarrow} \mathrm{GL}(\operatorname{Lie}(G))
$$


which for any superalgebra $A$ and $g \in G(A), x \in \operatorname{Lie}(G)(A)$

$$
\operatorname{Ad}_{A}(g) x={ }_{\operatorname{def}} G(i)(g) x G(i)(g)^{-1}
$$

$\operatorname{Ad}_{A}(g) x \in G(A(\epsilon))$ but since $G(p)$ is a group homomorphism, $\operatorname{Ad}_{A}(g) x \in$ $\operatorname{Lie}(G)(A)$.

$\mathrm{Ad}$ is a morphism in the category of group functors. So we can make the following definition:

Definition 3.6 Let $G$ be a supergroup functor. The adjoint action of $\operatorname{Lie}(G)$ on $\operatorname{Lie}(G)$ is defined as

$$
\operatorname{ad}=_{\operatorname{def}} \operatorname{Lie}(\operatorname{Ad}): \operatorname{Lie}(G) \longrightarrow \operatorname{Lie}(\operatorname{GL}(\operatorname{Lie}(G)))=\operatorname{End}(\operatorname{Lie}(G))
$$

Finally, the bracket on $\operatorname{Lie}(G)(A)$ is defined as

$$
[x, y]={ }_{\operatorname{def}} \operatorname{ad}(x) y, \quad x, y \in \operatorname{Lie}(G)(A) .
$$

The arguments in Ref. 42 II $\S 44.2,4.3$ apply with small changes to our case and prove that $[$,$] is a Lie bracket.$

\section{Example 3.3 $\mathrm{GL}_{m \mid n}$}

We want to see that in the case of $\mathrm{GL}_{m \mid n}$ the Lie bracket [, ] is the commutator. We have

$$
\begin{array}{ccc}
\operatorname{Ad}_{A}: \operatorname{GL}(A) & \longrightarrow & \mathrm{GL}\left(\operatorname{Lie}\left(\mathrm{GL}_{m \mid n}\right)\right)(A)=\mathrm{GL}\left(\mathrm{M}_{m \mid n}(A)\right) \\
g & \operatorname{Ad}(A)(g), \\
\operatorname{Ad}(g) x=g x g^{-1}, \quad x \in \mathrm{M}_{m \mid n}(A) .
\end{array}
$$

By definition we have: $\operatorname{Lie}\left(\operatorname{GL}\left(\mathfrak{g l}_{m \mid n}\right)\right)(A)=\left\{1+\epsilon \alpha / \alpha \in \operatorname{GL}\left(\mathfrak{g l}_{m \mid n}\right)(A)\right\}$ So we have, for $a, b \in \mathfrak{g l}_{m \mid n}(A) \cong \operatorname{Lie}\left(\operatorname{GL}_{m \mid n}\right)(A)=\left\{1+\epsilon a \mid a \in \mathfrak{g l}_{m \mid n}(A)\right\}$ :

$$
\operatorname{ad}(1+\epsilon a) b=(1+\epsilon a) b(1-\epsilon a)=b+(a b-b a) \epsilon=b+\epsilon[a, b] .
$$

Hence $\operatorname{ad}(1+\epsilon a)=\mathbb{1}+\epsilon \alpha(a)$, with $\alpha(a)=[a$,$] as we wanted to show.$

It is also clear that the same computation will hold for any closed subgroup of $\mathrm{GL}_{m \mid n}$.

Finally we have to address the issue of the the representability of the functor $\operatorname{Lie}(G)$. From the classical (non super) case, we know that it is not, 
in general, representable (see Ref. [42] II, 4, 4.8). The most useful example is when $G$ is a linear group, that is, a closed subgroup of $\mathrm{GL}_{n}$. Then $\operatorname{Lie}(G)$ is representable, and we will see that the same is true for the super case.

More generally, for any affine algebraic supergroup $G$, the associated functor $\operatorname{Lie}(G)$ can be shown to be representable. Theorem 3.1 characterizes $\operatorname{Lie}(G)$ geometrically as the tangent space at the identity. In the classical case, this result is a particular case of a more general one involving schemes, whose proof can be found in Ref. [42]. Since we are only concerned with affine algebraic supergroups, all we need is an extension to the super category of the (simpler) proof for affine algebraic groups. We find then convenient to write explicitly the proof of the following theorem, although the super extension in this particular case presented no difficulty.

Theorem 3.1 Let $k$ be a field and $G$ be an affine algebraic group, with $k[G]$ its coordinate superalgebra. As in Section [3.1, let $\mathcal{E}$ denote the counit in the superHopf algebra $k[G]$. We denote by $\mathbf{m}_{\mathcal{E}}=\operatorname{ker}(\mathcal{E})$ and by $\omega$ be the super vector space $\mathbf{m}_{\mathcal{E}} / \mathbf{m}_{\mathcal{E}}^{2}$. Then, $\operatorname{Lie}(G)$ is a representable functor and it is represented by $k[\omega]$ where

$$
k[\omega]=k\left[x_{1}, \ldots x_{p}, \xi_{1}, \ldots \xi_{q}\right]
$$

with $x_{1} \ldots x_{p}$, and $\xi_{1} \ldots \xi_{q}$ being even and odd indeterminates respectively, and $p \mid q$ is the superdimension of $\omega$.

Proof. We have to prove that $\operatorname{Lie}(G)(A)=\operatorname{hom}_{k-\operatorname{superalg}}(k[\omega], A)$. It is immediate to verify that

$$
\operatorname{hom}_{k-\operatorname{superalg}}(k[\omega], A) \cong \operatorname{hom}_{k-\operatorname{supermod}}(\omega, A) \cong\left(\omega^{*} \otimes A\right)_{0},
$$

hence it is enough to show that $\operatorname{Lie}(G)(A) \cong \operatorname{hom}_{k-\operatorname{supermod}}(\omega, A)$.

We will define a map $\rho: \operatorname{hom}_{k-\text { supermod }}(\omega, A) \rightarrow \operatorname{Lie}(G)(A)$ and then show that it is a bijection. Let $d \in \operatorname{hom}_{k-\text { supermod }}(\omega, A), d: \omega \longrightarrow A$. We first consider the following maps:

$$
\begin{aligned}
\phi: k[G] & \longrightarrow k \oplus \omega=k \oplus \mathbf{m}_{\mathcal{E}} / \mathbf{m}_{\mathcal{E}}^{2} \\
f & \mapsto\left(\mathcal{E}(f), f-\mathcal{E}(f)+\mathbf{m}_{\mathcal{E}}^{2}\right),
\end{aligned}
$$

and for each $d$

$$
\begin{aligned}
& d^{\prime}: k \oplus \omega \longrightarrow A(\epsilon) \\
& (s, t) \mapsto s+d(t) \epsilon .
\end{aligned}
$$


We define $\rho(d)$ as the composition $\rho(d)=d^{\prime} \circ \phi$. Then we have that

$$
G(p)(\rho(d))(f)=\mathcal{E}(f),
$$

so $\rho(d) \in \operatorname{ker} G(p)=\operatorname{Lie}(G)(A)$.

We want now to give the inverse $z: \operatorname{Lie}(G)(A) \rightarrow \operatorname{hom}_{k-\operatorname{supermod}}(\omega, A)$. Assume $\psi \in \operatorname{Lie}(G)(A)$. We can write: $\psi(f)=\mathcal{E}(f)+\epsilon f^{\prime}$. Now consider

$$
\begin{aligned}
\left.\psi\right|_{\mathbf{m}_{e}}: \mathbf{m}_{e} & \longrightarrow A(\epsilon) \\
f & \mapsto \epsilon f^{\prime}
\end{aligned}
$$

Observe that $\left.\psi\right|_{\mathbf{m}_{e}^{2}}=0$, hence going to the quotient we have a supermodule map $\tilde{\psi}: \omega \longrightarrow A(\epsilon), \tilde{\psi}(f)=f^{\prime} \epsilon$. Now define $z(\psi)(f)=f^{\prime}$. This is the inverse of $a$. The fact $z \cdot \rho=i d$ is straightforward. For $\rho \cdot z$ observe that given $\psi: k[G] \longrightarrow A(\epsilon)$, this can always be written as: $\psi(g)=\mathcal{E}(g)+g^{\prime \prime} \epsilon$. Observe that $g-\mathcal{E}(g) \in \mathbf{m}_{e}$ hence $\left.\psi\right|_{\mathbf{m}_{e}}(g-\mathcal{E}(g))=g^{\prime \prime} \epsilon$, now the result follows easily.

Corollary 3.1 Let $k$ be a field and $G$ be an affine algebraic supergroup. Then $\operatorname{Lie}(G)$ is a Lie superalgebra

We want to remark that $\omega=\mathbf{m}_{e} / \mathbf{m}_{e}^{2}$ can be regarded as the dual of the tangent space at the identity of the supergroup $G$. Theorem 3.1 hence states that such tangent space is the same as $\operatorname{Lie}(G)$, as it happens in the non super case.

Observation 3.1 Lie superalgebras as super vector spaces with a graded bracket.

Lie superalgebras were first introduced in physics [1] with a different definition. A Lie superalgebra is a super $\left(Z_{2}\right.$-graded $)$ vector space $\mathfrak{g}=\mathfrak{g}_{0}+\mathfrak{g}_{1}$ with a bilinear, graded operation

$$
\begin{aligned}
{[,]: \quad \mathfrak{g} \otimes \mathfrak{g} \longrightarrow } & \mathfrak{g} \\
X \otimes Y \longrightarrow & {[X, Y] }
\end{aligned}
$$

such that 
1. $[X, Y]=-(-1)^{p_{X} p_{Y}}[Y, X]$

2. $[X,[Y, Z]]+(-1)^{p_{X} p_{Y}+p_{X} p_{Z}}[Y,[Z, X]]+(-1)^{p_{X} p_{Z}+p_{Y} p_{Z}}[Z,[X, Y]]=0$

where $X, Y, Z$ are homogeneous elements of $\mathfrak{g}$ with parities $p_{X}, p_{Y}, p_{Z}$. This definition is used mostly when Lie superalgebras are treated independently of Lie supergroups [1], 16].

This definition of Lie superalgebra can be shown to be equivalent to Definition 3.3] in functorial terms. This is proven in Ref. [15], Corollary 1.7.3 pg 57, using the even rules principle. We will show how it works for the specific example $\mathfrak{g l}_{m \mid n}$ (Example 3.4).

We have then that in the super vector space $\omega^{*}$ there is a graded bracket $[$,$] and a Lie superalgebra structure in the sense mentioned above.$

\section{Example 3.4}

Let us consider the set of $(m+n) \times(m+n)$ matrices with arbitrary entries in $A$ denoted as $\overline{\mathfrak{g l}}_{m \mid n}(A)$. We also denote

$$
\begin{aligned}
I=1, \ldots m+n, & I=i \text { for } I=1, \ldots m \\
& I=n+\alpha \text { for } I=n+1, \ldots m+n .
\end{aligned}
$$

Let $\left\{E_{I J}\right\}$ be the standard basis of matrices with 1 in the place $I J$ and 0 everywhere else. An element $X \in \overline{\mathfrak{g l}}_{m \mid n}(A)$ can be written in terms of the standard basis

$$
X=X_{I J} E_{I J}=p_{i j} E_{i j}+q_{i \beta} E_{i \beta}+r_{\alpha j} E_{\alpha j}+s_{\alpha \beta} E_{\alpha \beta},
$$

where sum over repeated indices is understood and the parities of $p, q, r$ and $s$ are arbitrary.

We assign even degree to $E_{i j}$ and $E_{\alpha \beta}$ (block diagonal matrices) and odd degree to $E_{i \beta}$ and $E_{\alpha j}$ (block off diagonal matrices). This corresponds to even and odd linear maps. With this assignments $\overline{\mathfrak{g l}}_{m \mid n}(A)$ is a non commutative, associative superalgebra, and $\mathfrak{g l}_{m \mid n}(A)$ is its even part. It corresponds to the even linear maps or super vector space morphisms.

We can give it a super Lie algebra structure with the ordinary commutator of matrices among even elements or among an even and an odd one and the ordinary anticommutator of matrices among odd elements. Then, the even part of this Lie superalgebra is the Lie algebra $\mathfrak{g l}_{m \mid n}(A)$.

In general, giving a representable, Lie algebra valued functor is equivalent to give a super Lie algebra through the (anti)commutation rules of the generators. 


\section{Coadjoint orbits of supergroups.}

Let $G \subset \mathrm{GL}(m \mid n)$ be an algebraic Lie supergroup and $\mathfrak{g}=\operatorname{Lie}(G)$ the associated Lie superalgebra. Let $\mathfrak{g}^{*}$ be the functor $\mathfrak{g}^{*}(A)=\mathfrak{g}(A)^{*}$.

We want to define a coadjoint orbit of the supergroup $G$. Let $X_{0}$ be a geometric point of $\mathfrak{g}^{*}$, that is $X_{0} \in \mathfrak{g}^{*}(k) \cdot \mathfrak{g}(k)$ is an ordinary Lie algebra over $k$, and $\mathfrak{g}(k) \subset \mathfrak{g}(A)$ for any $A$ through the unit map of $A$. We consider the following functor,

$$
\begin{aligned}
\mathcal{C}_{X_{0}}:(\mathrm{salg}) & \longrightarrow(\text { sets }) \\
A & \longrightarrow\left\{\operatorname{Ad}_{g}^{*} X_{0}, \forall g \in G(A)\right\}=G(A) / H(A),
\end{aligned}
$$

where $H(A)$ is the stability group of $X_{0}$. Notice that it is necessary to choose a geometric point in order to have a functor.

The functor $\mathcal{C}_{X_{0}}$ is not, in general, representable. The problem arises already at the classical level. We can see it with an example. Let us consider the algebraic group $\mathrm{SL}_{n}$ over the complex numbers and its Lie algebra $\mathfrak{s l}_{n}$. Their functors of points are represented respectively by

$$
\begin{aligned}
\mathbb{C}\left[\mathrm{SL}_{n}\right] & =\mathbb{C}\left[x_{i j}\right] /(\operatorname{det}(x)-1), \quad i, j=1, \ldots n . \\
\mathbb{C}\left[\mathfrak{s l}_{n}\right] & =\mathbb{C}\left[x_{i j}\right] /(\operatorname{tr}(x))
\end{aligned}
$$

Let $X_{0}=\operatorname{diag}\left(l_{1} \ldots l_{n}\right) \in \mathfrak{s l}_{n}(\mathbb{C}), l_{i} \neq l_{j}$, for $i \neq j$. The coadjoint orbit of $X_{0}$ is an algebraic variety represented by

$$
\mathbb{C}\left[\mathcal{C}_{X_{0}}\right]=\mathbb{C}\left[x_{i j}\right] /\left(p_{1}-c_{1}, \cdots p_{l}-c_{l}\right),
$$

where $p_{i}=\operatorname{tr}\left(X^{i}\right)$ and $c_{i}=p_{i}\left(X_{0}\right)$.

An $A$-point of $\mathcal{C}_{X_{0}}$ is a morphism $\mathbb{C}\left[\mathcal{C}_{X_{0}}\right] \rightarrow A$ and it is given by a matrix $\left(a_{i j}\right) \in \mathfrak{g l}_{n}(A)$, such that $p_{k}\left(a_{i j}\right)=c_{k}, k=1 \ldots m+n$.

If the functor $\mathcal{C}_{X_{0}}^{r}$, defined as $\mathcal{C}_{X_{0}}$ but restricted to the category of commutative algebras were representable, any such matrix would be of the form $X=g X_{0} g^{-1}$, that is, conjugate to a diagonal one through an element $g \in \mathrm{SL}_{n}(A)$. But this is not necessarily true in an arbitrary algebra $A$.

In the classical case the functor of points of the coadjoint orbit is obtained as the sheafification (see Definition 4.1) of $\mathcal{C}_{X_{0}}^{r}$ (42] pg 341). We will see that the same is true for the super case. 
We will start by considering a functor

$$
\mathcal{F}:(\text { salg }) \longrightarrow \text { (sets). }
$$

This is a generalization of the concept of $\mathbb{Z}$-functor defined in [42 pg 9 .

$\mathcal{C}_{X_{0}}$ is an example of such a functor. Due to the equivalence of categories between (salg) and (sschemes aff) we can give equivalently the functor:

$$
\mathcal{F}:\left(\text { sschemes }_{\text {aff }}\right)^{\text {opp }} \longrightarrow \text { (sets). }
$$

(Abusing the notation, we use the same letter for both functors).

For each affine superscheme $X$ the restriction $\mathcal{F}_{X}$ of $\mathcal{F}$ to $\operatorname{Top}(X)$ defines a presheaf on $X(\operatorname{Top}(X)$ denotes the category of open sets in $X)$. A functor $\mathcal{F}:$ (salg) $\longrightarrow$ (sets) is said to be local if $\mathcal{F}_{X}$ is a sheaf for each $X \in$ (sschemes).

Equivalently, $\mathcal{F}$ is local if for any $A \in\left(\operatorname{salg}_{\mathrm{rf}}\right)$ and elements $f_{1} \ldots f_{s} \in A$ such that $\left(f_{1} \ldots f_{s}\right)=(1)$ we have the exact sequence:

$$
F(A) \stackrel{F\left(\alpha_{i}\right)}{\rightarrow} F\left(A_{f_{i}}\right) \stackrel{F\left(\alpha_{i j}\right)}{\rightrightarrows} F\left(A_{f_{i} f_{j}}\right)
$$

In particular, any representable functor is local.

Definition 4.1 Let us consider a functor $\mathcal{F}:(\mathrm{salg}) \longrightarrow$ (sets). We will denote by $\tilde{\mathcal{F}}$ the unique local functor such that for $f_{1} \ldots f_{s} \in A,\left(f_{1} \ldots f_{s}\right)=$ (1), $\tilde{\mathcal{F}}\left(A_{f_{i}}\right)=\mathcal{F}\left(A_{f_{i}}\right)$. $\tilde{\mathcal{F}}$ is called the sheafification of $\mathcal{F}$.

It is perhaps more natural to introduce the sheafification in terms of the presheaves $\mathcal{F}_{X}$. To any presheaf one can associate a sheaf that is the "closest" sheaf to the given presheaf; it is called its sheafification. Then $\tilde{\mathcal{F}}$ can be equivalently defined as the local functor obtained by doing the sheafification of the presheaves $\mathcal{F}_{X}$ for all $X \in$ (sschemes).

Observation 4.1. If $\mathcal{P}$ is a subfunctor of a local functor $\mathcal{F}$ and for all $R \in(\mathrm{salg})$ there exist $f_{1} \ldots f_{r} \in R^{o}$ such that $\left(f_{1} \ldots f_{r}\right)=(1)$ and $\mathcal{P}\left(R_{f_{i}}\right)=$ $\mathcal{F}\left(R_{f_{i}}\right)$ then $\tilde{\mathcal{P}}=\mathcal{F}$. ( $R$ is viewed as an $R_{0}$ module).

Our strategy to determine the functor of the coadjoint orbits of supergroups will be to find a representable (hence local) functor and to prove that it is the sheafification of (2).

We need first some notions about the invariant polynomials of a superalgebra. 


\subsection{Invariant polynomials}

For the rest of the section we take $k=\mathbb{C}$. The Cartan-Killing form of a super Lie algebra $\mathfrak{g}$ is a natural trasformation: $B: \mathfrak{g} \times \mathfrak{g} \longrightarrow \mathfrak{g}$,

$$
B_{A}(X, Y)=\operatorname{str}\left(\operatorname{ad}_{X} \operatorname{ad}_{Y}\right) \quad X, Y \in \mathfrak{g}(A) .
$$

$B_{A}$ is an invariant, supersymmetric bilinear form. In the following, we will consider simple Lie superalgebras whose Cartan-Killing form is non degenerate, namely $\mathfrak{s l}_{m \mid n}$ with $m \neq n, \mathfrak{o s p}_{m \mid n}$ with $\frac{m}{2}-\frac{n}{2} \neq 1$ ( $m, n$ even), $\mathfrak{f}_{4}$ and $\mathfrak{g}_{3}$ [16.

There is a natural isomorphism between the functors $\mathfrak{g}$ and $\mathfrak{g}^{*}$ such that to each object $A$ in $\left(\operatorname{salg}_{\text {rf }}\right)$ assigns a morphism

$$
\begin{aligned}
\varphi_{A}: \mathfrak{g}(A) & \longrightarrow \mathfrak{g}^{*}(A) \\
X & \longrightarrow \varphi_{A}(X) \text { with } \varphi_{A}(X)(Y)=B_{A}(X, Y) .
\end{aligned}
$$

This isomorphism gives also an isomorphism between the superalgebras representing both functors, $\mathbb{C}[\mathfrak{g}] \simeq \mathbb{C}\left[\mathfrak{g}^{*}\right]$ and intertwines the adjoint and coadjoint representation, so the adjoint orbits are the same as the coadjoint orbits. From now on we will use the algebra $\mathbb{C}[\mathfrak{g}]$.

Let $G$ be an affine algebraic supergroup, subfunctor of $\operatorname{GL}(m \mid n)$, with super Lie algebra $\mathfrak{g}$. We say that $p \in \mathbb{C}[\mathfrak{g}]$ is an invariant polynomial if for any $A$-point $x: \mathbb{C}[\mathfrak{g}] \rightarrow A$ of $\mathfrak{g}$ and $g: \mathbb{C}[G] \rightarrow A$ of $G$ we have that

$$
x(p)=\operatorname{Ad}_{A}(g) x(p) .
$$

The invariant polynomials are a subalgebra of $\mathbb{C}[\mathfrak{g}]$. Contrary to what happens in the classical case, this algebra may be not finitely generated [11, 44. This is the case for the algebra of invariant polynomials on $\mathfrak{g l}_{m \mid n}$. The generators can be taken to be the supertraces of arbitrary order, $\operatorname{str}\left(X^{k}\right)$, which are independent. The invariant polynomials in the reduced Lie algebra, $\mathfrak{g l}_{m} \times \mathfrak{g l}_{n}$ are generated by $\operatorname{traces} \operatorname{tr}\left(X^{k}\right)$ with $k=1, \ldots m+n$, since higher order traces can be expressed in terms of the first $m+n$ ones.

\subsection{The coadjoint orbits of a supergroup as algebraic supervarieties}

For a regular, semisimple element $X_{0}$ of $\mathfrak{g}(\mathbb{C})$, its orbit under the adjoint action of the group $G(\mathbb{C})$ is an algebraic variety defined by the values of 
the homogeneous Chevalley polynomials $p_{1}, \ldots p_{l}$, where $l$ is the rank of the group. We will see that the supersymmetric extensions of these polynomials define the adjoint orbit of the supergroup. More specifically, we have the following

Theorem 4.1 Let $\hat{p}_{1}, \ldots \hat{p}_{l}$ be polynomials on a simple Lie superalgebra of the type $\mathfrak{s l}_{m \mid n}$ or $\mathfrak{o s p}_{m \mid n}$ with the following properties:

1. They are invariant polynomials under the adjoint action (4).

2. Let $p_{1}, \ldots p_{l}$ be the projections of $\hat{p}_{1}, \ldots \hat{p}_{l}$ onto the reduced algebra $\mathbb{C}\left[\mathfrak{g}^{\circ}\right]=$ $\mathbb{C}[\mathfrak{g}] / \mathcal{I}^{\text {odd }}$. The ideal of the orbit of a regular, semisimple element $X_{0} \in \mathfrak{g}(\mathbb{C})$ with distinct eigenvalues, is $\mathcal{J}=\left(p_{1}-c_{1}, \ldots p_{l}-c_{l}\right), c_{i}=p_{i}\left(X_{0}\right)$. So the orbit of $X_{0}$ is an algebraic variety whose functor is represented by

$$
\mathbb{C}\left[\mathfrak{g}^{\circ}\right] / \mathcal{J}
$$

Then the sheafification of the functor $\mathcal{C}_{X_{0}}$ (2) is representable and is represented by

$$
A_{X_{0}}=\mathbb{C}[\mathfrak{g}] / \mathcal{I}
$$

with $\mathcal{I}=\left(\hat{p}_{1}-c_{1}, \ldots \hat{p}_{l}-c_{l}\right)$.

Proof. Let us denote by $F:\left(\operatorname{salg}_{\mathrm{rf}}\right) \rightarrow$ (sets) the functor represented by $A_{X_{0}}$ in (15). It is clear that $\mathcal{C}_{X_{0}}$ is a subfunctor of $F$, since for any superalgebra $R$, an element of $\mathcal{C}_{X_{0}}(R)=\left\{g X_{0} g^{-1}, g \in G(R)\right\}$ is given by a matrix in $\mathfrak{g}(R)$,

$$
M:\left(\begin{array}{cc}
p_{i j} & q_{i \beta} \\
r_{\alpha j} & s_{\alpha \beta}
\end{array}\right)
$$

whose entries satisfy $p_{1}-c_{1}=0, \ldots p_{l}-c_{l}=0$, and then defines a homomorphism

$$
M: A_{X_{0}} \longrightarrow R .
$$

In view of the Observation (4.1) we just have to show that for $f_{1} \ldots f_{s} \in R^{o}$, $\left(f_{1} \ldots f_{s}\right)=(1), F\left(R_{f_{i}}\right)=\mathcal{C}_{X_{0}}\left(R_{f_{i}}\right)$.

Let $f \in R^{\circ}$. By the previous observation there is an obvious injective map $\mathcal{C}_{X_{0}}\left(R_{f}\right) \rightarrow F\left(R_{f}\right)$. We have to show that the $f_{i}$ can be chosen in such a way that the map is also surjective.

This means that we need to prove that given $W \in \mathcal{C}_{X_{0}}(R)$, there exist $f \in R^{\circ}$ and $g \in G\left(R_{f}\right)$ such that

$$
g W g^{-1}=D, \quad \text { or } \quad g W=D g
$$


where $D$ is a diagonal matrix diagonal. Later on we will prove that $D=X_{0}$.

We consider first a superalgebra $R$ is a free superalgebra in the odd generators. We decompose the matrices in (6) as sums of matrices whose elements are homogeneous in the odd variables

$$
\left(g_{0}+g_{1}+\cdots\right)\left(W_{0}+W_{1}+\cdots\right)=\left(D_{0}+D_{1}+\cdots\right)\left(g_{0}+g_{1}+\cdots\right) \text {. }
$$

Then we can compare elements of the same degree obtaining

$$
g_{0} W_{n}+g_{1} W_{n-1}+\cdots g_{n} W_{0}=D_{0} g_{n}+D_{1} g_{n-1}+\cdots+D_{n} g_{0} .
$$

We will prove the result by induction. For $n=0$ we have

$$
g_{0} W_{0}=D_{0} g_{0}
$$

But this is the classical result, with $D_{0}=X_{0}$. By the hypothesis 2. of the theorem, $\left(p_{1}-c_{1}, \ldots p_{l}-c_{l}\right)$ is the ideal of the reduced orbit, so when we restrict to the category of commutative algebras we know that $F$ is the sheafification of $\mathcal{C}_{X_{0}}$ and so it is represented by $\mathbb{C}\left[\mathfrak{g}^{\circ}\right] /\left(p_{1}-c_{1} \ldots p_{l}-c_{l}\right)$.

This means that there exist $f \in R^{\circ}, g_{0} \in G\left(R^{\circ}\right)$, such that $g_{0} W_{0} g_{0}^{-1}=$ $D_{0}$. Moreover, the classical results guarantees that one can choose $f_{1} \ldots f_{r}$ among all possible $f$ 's in such a way that the ideal that they generate in $R^{\circ}$, $\left(f_{1} \ldots f_{r}\right)=(1)=R^{\circ}$.

The induction proof is based on an argument given in Ref. [11], page 117. We assume that the result is true up to order $n-1$. Then we multiply (7) by $g_{0}^{-1}$ to the right. Using (8) we obtain

$$
X_{0} g_{n} g_{0}^{-1}-g_{n} g_{0}^{-1} X_{0}+D_{n}=K_{n},
$$

where $K_{n}$ is a known matrix

$$
K_{n}=-D_{1} g_{n-1} g_{0}^{-1}-D_{2} g_{n-2} g_{0}^{-1}-\cdots+g_{0} W_{n-1} g_{0}^{-1}+\cdots+g_{n-1} W_{1} g_{0}^{-1}
$$

and $D_{n}$ is a diagonal matrix. The matrix $X_{0} g_{n} g_{0}^{-1}-g_{n} g_{0}^{-1} X_{0}$ has only elements outside the diagonal, and if $\left(\lambda_{i}\right)$ are the eigenvalues of $X_{0}$ we have that the entry $(i j)$ is given by

$$
\left(X_{0} g_{n} g_{0}^{-1}-g_{n} g_{0}^{-1} X_{0}\right)_{i j}=\left(\lambda_{i}-\lambda_{j}\right)\left(g_{n} g_{0}^{-1}\right)_{i j} .
$$

Then, $g_{n}$ and $D_{n}$ can be computed from (9) provided $\lambda_{i} \neq \lambda_{j}$. 
To finish the proof we will have to show that $D_{n}=0$ for $n \geq 0$. Let us consider the case of $\mathfrak{s l}(m \mid n)$. Then the invariant polynomials $p_{i} \in \mathbb{C}[\mathfrak{s l}(m \mid n)]$ that we have to consider are

$$
p_{i}(M)=\operatorname{str} M^{i}, \quad i=1, \ldots m+n .
$$

We want to prove that for a diagonal matrix $D=D_{0}+D_{1}+\cdots$, if

$$
p_{i}(D)=p_{i}\left(D_{0}\right),
$$

then $D=D_{0}$. Let

$$
D=\operatorname{diag}\left(\lambda_{1}+\lambda_{1}^{\prime}, \lambda_{2}+\lambda_{2}^{\prime}, \ldots \lambda_{m+n}+\lambda_{m+n}^{\prime}\right),
$$

with $\lambda_{i}^{\prime}$ contains all the terms in the odd variables. Then (10) implies the following homogeneous system:

$$
\left(\begin{array}{cccccc}
1 & \cdots & 1 & -1 & \cdots & -1 \\
\lambda_{1} & \cdots & \lambda_{m} & -\lambda_{m+1} & \cdots & -\lambda_{m+n} \\
\cdots & & & & & \\
\lambda_{1}^{m+n} & \cdots & \lambda_{m}^{m+n} & -\lambda_{m+1}^{m+n} & \cdots & -\lambda_{m+n}^{m+n}
\end{array}\right)\left(\begin{array}{c}
\lambda_{1}^{\prime} \\
\lambda_{2}^{\prime} \\
\cdots \\
\lambda_{m+n}^{\prime}
\end{array}\right)
$$

which can have non trivial solution solution if the determinant

$$
\operatorname{det}\left(\begin{array}{cccccc}
1 & \cdots & 1 & -1 & \cdots & -1 \\
\lambda_{1} & \cdots & \lambda_{m} & -\lambda_{m+1} & \cdots & -\lambda_{m+n} \\
\cdots & & & & & \\
\lambda_{1}^{m+n} & \cdots & \lambda_{m}^{m+n} & -\lambda_{m+1}^{m+n} & \cdots & -\lambda_{m+n}^{m+n}
\end{array}\right)=(-1)^{n m} \prod_{i>j}\left(\lambda_{i}-\lambda_{j}\right)
$$

is different from zero (Vandermonde determinant). So we have our result if all the eigenvalues are different.

In the case of $\mathfrak{o s p}_{m \mid n}$, the relevant polynomials are of the form $\operatorname{str} M^{2 i}$, so the result can be reproduced without difficulty.

In the case we consider a superalgebra $R / J$ with $J$ an ideal, it is enough to look at the images of the $\left(f_{1}, \cdots f_{r}\right)$ under the projection $F / J$.

\section{Deformation quantization of coadjoint or- bits of supergroups.}

Let $k=\mathbb{C}$. We start with the definitions of Poisson superalgebra and its deformation. 
Definition 5.1 Let $A$ be a commutative superalgebra. We say that $A$ is a Poisson superalgebra if there exists a linear map (Poisson superbracket)

$$
\begin{aligned}
\{,\}: A \otimes A & \rightarrow \\
f \otimes g & \longrightarrow\{f, g\}
\end{aligned}
$$

such that

1. $\{a, b\}=-(-1)^{p_{a} p_{b}}\{b, a\}$

2. $\{a,\{b, c\}\}+(-1)^{p_{a} p_{b}+p_{a} p_{c}}\{b,\{c, a\}\}+(-1)^{p_{a} p_{c}+p_{b} p_{c}}\{c,\{a, b\}\}=0$

3. $\{a, b c\}=\{a, b\} c+(-1)^{p_{a} p_{b}} b\{a, c\}$

where $a, b, c$ are homogeneous elements of $A$ with parities $p_{a}, p_{b}, p_{c}{ }^{4}$.

Let $\mathfrak{g}$ be a Lie superalgebra with Lie superbracket

$$
\left[X_{I}, X_{J}\right]=c_{I J}^{K} X_{K}
$$

for a certain homogeneous basis $\left\{X_{I}\right\}_{I=1}^{s+r}$ with the first $s$ vectors even and the last $r$ odd. Then $\mathbb{C}\left[\mathfrak{g}^{*}\right] \simeq \mathbb{C}\left[x_{1}, \ldots x_{s}, x_{s+1}, \cdots x_{s+r}\right]$ has a Poisson superalgebra structure with superbracket given by

$$
\left\{x_{I}, x_{J}\right\}=\sum_{K} x_{K}\left(\left[X_{I}, X_{J}\right]\right) x_{K}=\sum_{K} c_{I J}^{K} x_{K}
$$

and extended to the whole algebra by property 3. of Definition 5.1 .

Let $\mathfrak{g}$ be one of the Lie algebras considered in Theorem 4.1, which have a Cartan-Killing form, so $\mathfrak{g} \simeq \mathfrak{g}^{*}$ and $\mathbb{C}[\mathfrak{g}] \simeq \mathbb{C}\left[\mathfrak{g}^{*}\right]$. Let $A=\mathbb{C}[\mathfrak{g}] / \mathcal{I}$ be the algebra associated to the superorbit of a geometric element $X_{0}$ with the conditions of Theorem 4.1. Then $A$ is also a Poisson superalgebra with the Poisson superbracket induced by the one in $\mathbb{C}[\mathfrak{g}]$. This follows from the derivation property 3 . in Definition 5.1 and the fact that $\hat{p}_{i}$ are invariant polynomials.

In this section we want to construct a deformation quantization of the superalgebra representing the orbit of a supergroup. We will extend the method used in Refs. 28, 29] for the classical (non super) case.

Definition 5.2 Given a Poisson superalgebra A, a formal deformation (or deformation quantization) of $A$ is an associative non commutative superalgebra algebra $A_{h}$ over $\mathbb{C}[[h]]$, where $h$ is a formal parameter, with the following properties:

\footnotetext{
${ }^{4}$ We want to remark that with this definition we have only even Poisson brackets.
} 
1. $A_{h}$ is isomorphic to $A[[h]]$ as a $\mathbb{C}[[h]]$-module.

2. The multiplication $*_{h}$ in $A_{h}$ reduces $\bmod (h)$ to the one in $A$.

3. $\tilde{a} *_{h} \tilde{b}-\tilde{b} *_{h} \tilde{a}=h\{a, b\} \bmod \left(h^{2}\right)$, where $\tilde{a}, \tilde{b} \in A_{h}$ reduce to $a, b \in A$ $\bmod (h)$ and $\{$,$\} is the Poisson superbracket in A$.

Let $\mathfrak{g}$ be a Lie superalgebra and $\mathfrak{g}_{h}$ the Lie superalgebra over $\mathbb{C}[[h]]$ obtained by multiplying the Lie bracket of $\mathfrak{g}$ by the formal parameter $h$. Let us denote by $U_{h}$ the universal enveloping algebra of $\mathfrak{g}_{h}$ ([1] pg. 279). As in the classical case, it is easy to prove that the associative, non commutative superalgebra $U_{h}$ is a deformation quantization of $\mathbb{C}\left[\mathfrak{g}^{*}\right]$.

Let $\mathfrak{g}$ be a Lie superalgebra over $\mathbb{C}$ of the type considered in Theorem 4.1. By property 1 in Definition 5.2 , there exists an isomorphism of $\mathbb{C}[[h]]$ modules $\left.\psi: \mathbb{C}\left[\mathfrak{g}^{*}\right][[h]]\right] \rightarrow U_{h}$. We want to prove that $\psi$ can be chosen in such a way that there exists an ideal $\mathcal{I}_{h} \subset U_{h}$ such that $\psi(\mathcal{I})=\mathcal{I}_{h}{ }^{5}$, and the map induced on the quotients $\psi_{h}: \mathbb{C}\left[\mathfrak{g}^{*}\right] / \mathcal{I}[[h]] \rightarrow U_{h} / I_{h}$ is an isomorphism of $\mathbb{C}[[h]]$-modules. We have then that the following diagram

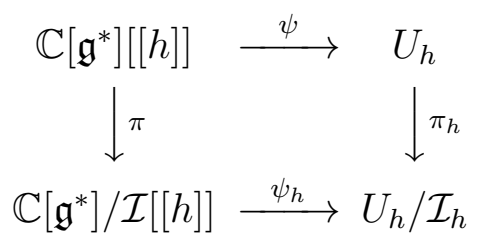

with $\pi$ and $\pi_{h}$ the canonical projections, commutes.

We list first some known results about the enveloping algebra of $\mathfrak{g}, U(\mathfrak{g})$ 11. We denote by $S(\mathfrak{g})$ the algebra of super symmetric tensors on $\mathfrak{g}$. We have that $S(\mathfrak{g}) \simeq \mathbb{C}\left[\mathfrak{g}^{*}\right]$. As before, $\left\{X_{I}\right\}_{I=1}^{s+r}$ denotes a homogeneous basis of $\mathfrak{g}$ as vector superspace. Let $\tau: \mathbb{C}\left[\mathfrak{g}^{*}\right] \rightarrow U(\mathfrak{g})$ be the supersymmetrizer map

$$
\tau\left(x_{I_{1}} \cdots x_{I_{p}}\right)=\frac{1}{p !} \sum_{s \in S_{p}}(-1)^{\bar{\sigma}(s)} X_{s\left(I_{1}\right)} \otimes \cdots \otimes X_{s\left(I_{p}\right)}
$$

where $S_{p}$ is the group of permutations of order $p$ and $\bar{\sigma}(s)$ is the sign arising when performing the permutation $X_{I_{1}} \otimes \cdots \otimes X_{I_{p}} \stackrel{s}{\longrightarrow} X_{s\left(I_{1}\right)} \otimes \cdots \otimes X_{s\left(I_{p}\right)}$ as if the homogeneous elements $X_{I_{j}}$ where supercommuting. $\tau$ is an isomorphism of $\mathfrak{g}$-modules. Let $\mathbb{C}[\mathfrak{g}]^{\mathfrak{g}}$ denote the set of polynomials invariant under

\footnotetext{
${ }^{5}$ the ideal $\mathcal{I}$ is understood here as an ideal of $\mathbb{C}\left[\mathfrak{g}^{*}\right][[h]]$
} 
the adjoint action of $\mathfrak{g}$ (in particular $\hat{p}_{i} \in \mathbb{C}[\mathfrak{g}]^{\mathfrak{g}}$ ). Then $\tau$ induces an isomorphism of $\mathfrak{g}$-modules:

$$
\mathbb{C}[\mathfrak{g}]^{\mathfrak{g}} \simeq Z(U(\mathfrak{g}))
$$

where $Z(U(\mathfrak{g}))$ is the center of $U(\mathfrak{g})$

Let $\mathcal{I}=\left(p_{1}-c_{1}, \ldots p_{l}-c_{l}\right)$ as in Theorem 4.1. We set

$$
\mathcal{I}_{h}=\left(P_{1}-c_{1}(h) \ldots P_{l}-c_{l}(h)\right) \subset U_{h}, \quad P_{i}=\tau\left(\hat{p}_{i}\right), \quad c_{k}(0)=c_{k},
$$

with $c_{i}(h) \in \mathbb{C}[[h]]$.

Theorem 5.1 In the settings of Theorem 4.1. $U_{h} / \mathcal{I}_{h}$ is a deformation quantization of $\mathbb{C}\left[\mathfrak{g}^{*}\right] / \mathcal{I}$.

Proof The only property in Definition 5.2 which is not immediate is property a., that is, the fact of $U_{h} / \mathcal{I}_{h} \simeq \mathbb{C}\left[\mathfrak{g}^{*}\right] / \mathcal{I}[[h]]$ as $\mathbb{C}[[h]]$-modules. The proof can be almost translated from the classical case in Ref. [28]. Let $\left\{x_{I_{1}}, \ldots, x_{I_{k}}\right\}_{\left(I_{1}, \ldots, I_{k}\right) \in S}$ be a basis of $\mathbb{C}\left[\mathfrak{g}^{*}\right] / I$ as $\mathbb{C}$-module, where $S$ is a fixed set of multiindices appropriate to describe the basis. In particular, we can take them such that $I_{1} \leq \cdots \leq I_{k}$. Proving that the monomials $\mathcal{B}=\left\{X_{I_{1}} \cdots X_{I_{k}}\right\}_{\left(I_{1}, \ldots, I_{k}\right) \in S}$ are a basis for $U_{h} / I_{h}$ will be enough. The proof that $\mathcal{B}$ is a generating set for $U_{h} / I_{h}$ is identical to the proof of Proposition 3.13 in Ref. [28] and we will not repeat it here. For the linear independence, we have to show that there is no relation among them. Suppose that $G \in \mathcal{I}_{h}$ is such relation,

$$
G=G_{0}+G_{1} h+\cdots, \quad G_{i} \in \operatorname{span}_{\mathbb{C}}\left\{X_{I_{1}} \cdots X_{I_{k}}\right\}_{\left(I_{1} \ldots I_{k}\right) \in S} .
$$

Assume $G_{i}=0, i<r, G_{r} \neq 0$ so we can write $G=h^{r} F$, with

$$
F=F_{0}+h F_{1}+\cdots, \quad F_{0} \neq 0 .
$$

We need to prove the non trivial fact that if $h F \in \mathcal{I}_{h}$ then $F \in \mathcal{I}_{h}$. We will denote by a capital letter, say $P$, an element in $U_{h}$, by $\hat{p}$ its projection onto $\mathbb{C}\left[\mathfrak{g}^{*}\right]$ and by $p$ its further projection onto $\mathbb{C}\left[\mathfrak{g}^{\circ *}\right]$. Assume that

$$
h F=\sum_{i} A_{i}\left(P_{i}-c_{i}(h)\right)
$$

with $c_{i}(h)=c_{i}+c_{i}^{1} h+\ldots c_{n} h^{n}$. 
Then, reducing mod $h$ (taking $h=0$ ) we obtain

$$
0=\sum_{i} \hat{a}_{i}\left(\hat{p}_{i}-c_{i}\right)
$$

Setting all the odd variables to 0, we have that (13) implies that there exist $b_{i j}$, antisymmetric in $i$ and $j$ such that

$$
a_{i}=\sum_{j} b_{i j}\left(p_{j}-c_{j}\right)
$$

(see for example Ref. [45], pg 81, or lemma 3.8 of Ref. [28]), provided that the differentials $d p_{i}$ are independent on the orbit (condition which is satisfied 46] in our case). The generalization of this property to the supersymmetric case deserves special treatment, but the proof is rather technical and we will do it separately in Lemma 5.1. Assuming that this is true, then there exist $\hat{b}_{i j}$, antisymmetric in $i$ and $j$ such that

$$
\hat{a}_{i}=\sum_{j} \hat{b}_{i j}\left(\hat{p}_{j}-c_{j}\right)
$$

It is easy to convince oneself that this equation can be lifted to $U_{h}$, so there exists $A_{i}^{\prime}$ and $B_{i j}$ antisymmetric in $i$ and $j$ such that

$$
A_{i}^{\prime}=\sum_{j} B_{i j}\left(P_{j}-c_{j}(h)\right)
$$

with $A_{i}=A_{i}^{\prime} \bmod h$, since they both project to $a_{i}$, i.e. $A_{i}=A_{i}^{\prime}+h C_{i}$. If one substitutes in (12):

$$
h F=h \sum_{i} C_{i}\left(P_{i}-c_{i}(h)\right)
$$

since $\sum_{j} B_{i j}\left(P_{j}-c_{j}(h)\right)\left(P_{i}-c_{i}(h)\right)=0$ (the $B_{i j}$ 's are antisymmetric). Hence we get the fact: $h F \in \mathcal{I}_{h}$ then $F \in \mathcal{I}_{h}$.

So being $F \in \mathcal{I}_{h}$, we can reduce (11) $\bmod h$,

$$
f=\sum a_{i}\left(\hat{p}_{i}-c_{i}\right)
$$

But $f$ would represent a non trivial relation among the monomials $\left\{x_{I_{1}} \cdots x_{I_{k}}\right\}_{\left(I_{1} \ldots I_{k}\right) \in S}$ in $\mathbb{C}\left[\mathfrak{g}^{*}\right] / \mathcal{I}$, which is a contradiction, so the linear independence is proven. 
Lemma 5.1 . Let $A$ be the free commutative superalgebra over $\mathbb{C}$ generated by $M$ even variables $x_{1} \ldots x_{M}$ and $N$ odd variables $\xi_{1} \ldots \xi_{N}$. Let $q_{1} \ldots q_{n}$ even polynomials in $A$ and denote by $q_{1}^{\circ}, \ldots q_{n}^{\circ}$ their projections onto $A^{\circ}$. Assume that the $q_{i}^{\circ}$ 's satisfy the following property:

If $\sum_{i} q_{i}^{\circ} f_{i}^{\circ}=0$ for some $f_{i}^{\circ} \in A^{\circ}$, then there exist $F_{i j}^{\circ} \in A^{\circ}$ such that $f_{i}^{\circ}=\sum_{j} F_{i j}^{\circ} q_{j}^{\circ}$, with $F_{i j}^{\circ}=-F_{j i}^{\circ}, i, j=1 \ldots n$.

Then, if $\sum_{i} q_{i} f_{i}=0$ for some $f_{i} \in A$, there exist $F_{i j} \in A$ such that $f_{i}=$ $\sum_{j} F_{i j} q_{j}$, with $F_{i j}=-F_{j i}, i, j=1 \ldots n$.

Proof. We write

$$
\begin{aligned}
f_{i} & =\sum_{k=0}^{N} \sum_{1 \leq \alpha_{1}<\ldots \alpha_{k} \leq N} f_{i}^{\alpha_{1} \ldots \alpha_{k}} \xi_{\alpha_{1}} \cdots \xi_{\alpha_{k}} \\
q_{i} & =\sum_{l=0}^{N} \sum_{1 \leq \beta_{1}<\ldots \beta_{l} \leq N} q_{i}^{\beta_{1} \ldots \beta_{l}} \xi_{\beta_{1}} \cdots \xi_{\beta_{l}} .
\end{aligned}
$$

Since $q_{i}$ are even functions we have that $l$ is always even number (that is, the components with $l$ odd are zero). The terms for $k=0$ or $l=0$ correspond to $f_{i}^{\circ}$ and $q_{i}^{\circ}$ respectively.

The condition $\sum_{i=1}^{n} f_{i} q_{i}=0$ reads in components

$$
\sum_{i=1}^{n} \sum_{s=0}^{p} \sum_{1 \leq k_{1}<\cdots<k_{s} \leq p}(-1)^{\ell\left(k_{1}, \ldots k_{s}, 1, \ldots \hat{k}_{1}, \ldots \hat{k}_{s}, \ldots p\right)} f_{i}^{\alpha_{k_{1}} \cdots \alpha_{k_{s}}} q_{i}^{\alpha_{1} \cdots \hat{\alpha}_{k_{1}} \cdots \hat{\alpha}_{k_{s}} \cdots \alpha_{p}}=0
$$

for $p=1, \ldots N$ and where $\ell\left(k_{1}, \ldots k_{s}, 1, \ldots \hat{k}_{1}, \ldots \hat{k}_{s}, \ldots p\right)$ is the length of the permutation $\left(k_{1}, \ldots k_{s}, 1, \ldots \hat{k}_{1}, \ldots \hat{k}_{s}, \ldots p\right)$. For $p=0$ we have

$$
\sum_{i=1}^{n} f_{i}^{\circ} q_{i}^{\circ}=0
$$

We will show the lemma by induction. Let $\mathcal{I}_{m}^{\text {odd }}$ be the ideal in $A$ generated by the products

$$
\mathcal{I}_{m}^{\text {odd }}=\left(\xi_{\alpha_{1}} \cdots \xi_{\alpha_{m+1}}, \ldots, \xi_{1} \cdots \xi_{N}\right),
$$


and consider the projections $\pi_{m}: A \rightarrow A / \mathcal{I}_{m}^{\text {odd }}$. Assume that there exists

$$
F_{i j}^{m}=\sum_{r=0}^{m} F_{i j}^{\delta_{1} \cdots \delta_{r}} \xi_{\delta_{1}} \cdots \xi_{\delta_{r}},
$$

with $F_{i j}^{m}=-F_{j i}^{m}$, such that

$$
\pi_{s}\left(f_{i}\right)=\pi_{s}\left(F_{i j}^{m} p_{j}\right)
$$

for all $s \leq m$. (To simplify the notation the sum over reapeated indices $i$ and $j$ will be understood). For $m=0$ this is the classical condition, that is guaranteed by (15) and the hypothesis of the theorem. We must show that there exist $F_{i j}^{\delta_{1} \cdots \delta_{m+1}}$ such that

$$
F_{i j}^{m+1}=\sum_{r=0}^{m+1} F_{i j}^{\delta_{1} \cdots \delta_{r}} \xi_{\delta_{1}} \cdots \xi_{\delta_{r}}
$$

satisfies

$$
\pi_{s}\left(f_{i}\right)=\pi_{s}\left(F_{i j}^{m+1} p_{j}\right)
$$

for all $s \leq m+1$. Since $\pi_{N}$ is the identity, we will have our result.

Let us write the induction hypothesis (16) in components,

$$
f_{i}^{\alpha_{1} \cdots \alpha_{s}}=\sum_{r=0}^{s} \sum_{1 \leq l_{1}<\cdots l_{r} \leq s}(-1)^{\ell\left(l_{1} \ldots l_{r}, 1, \ldots \hat{l}_{1}, \ldots \hat{l}_{r}, \ldots s\right)} F_{i j}^{\alpha_{l_{1}} \ldots \alpha_{l_{r}}} q_{j}^{\alpha_{1} \ldots \hat{\alpha}_{l_{1}} \ldots \hat{\alpha}_{l_{r}} \ldots \alpha_{s}},
$$

with $s \leq m$. We substitute this expression for $s \leq m$ in (14). Taking $p=m+1$ we have

$$
\begin{aligned}
& f_{i}^{\alpha_{1} \ldots \alpha_{m+1}} q_{i}^{0}+\sum_{s=0}^{m} \sum_{1 \leq k_{1}<\cdots<k_{s} \leq m+1} \sum_{r=0}^{s} \sum_{k_{1} \leq k_{l_{1}}<\cdots<k_{l_{r}} \leq k_{s}} \\
& (-1)^{\ell\left(k_{1}, \ldots k_{s}, 1, \ldots \hat{k}_{1}, \ldots \hat{k}_{s}, \ldots m+1\right)}(-1)^{\ell\left(k_{l_{1}} \ldots k_{l_{r}} k_{1} \ldots \hat{k}_{l_{1}} \ldots \hat{k}_{l_{r}} \ldots k_{s}\right)} \\
& F_{i j}^{\alpha_{k_{l_{1}}} \ldots \alpha_{k_{l}}} q_{j}^{\alpha_{k_{1}} \ldots \hat{\alpha}_{k_{l_{1}}} \ldots \hat{\alpha}_{k_{l_{r}}} \ldots \alpha_{k_{s}}} q_{i}^{\alpha_{1}, \ldots \hat{\alpha}_{k_{1}} \ldots \hat{\alpha}_{k_{s}} \ldots \alpha_{m+1}}=0
\end{aligned}
$$

We distinguish two kinds of terms in (18), the ones that are multiplied by $q_{i}^{\circ}$ and the ones that are not. A generic term that does not contain $q_{i}^{\circ}$ is of the form 


$$
\begin{aligned}
& (-1)^{\ell\left(k_{1}, \ldots k_{s}, 1, \ldots \hat{k}_{1}, \ldots \hat{k}_{s}, \ldots m+1\right)}(-1)^{\ell\left(k_{l_{1}} \ldots k_{l_{r}} k_{1} \ldots \hat{k}_{l_{1}} \ldots \hat{k}_{l_{r}} \ldots k_{s}\right)} \\
& F_{i j}^{\alpha_{k_{l_{1}}} \ldots \alpha_{k_{l_{r}}}} q_{j}^{\alpha_{k_{1}} \ldots \hat{\alpha}_{k_{l_{1}}} \ldots \hat{\alpha}_{k_{l_{r}}} \ldots \alpha_{k_{s}}} q_{i}^{\alpha_{1}, \ldots \hat{\alpha}_{k_{1}} \ldots \hat{\alpha}_{k_{s}} \ldots \alpha_{m+1}}
\end{aligned}
$$

with $r \neq s$ and $s \neq m+1$. We are going to see that these terms cancel. The reason is that for each term as (19) there exists an identical term where $q_{i}$ is interchanged with $q_{j}$. Then, they cancel because of the antisymmetry of $F_{i j}$. We first consider the following set of indices

$$
\left\{k_{1}^{\prime}, \ldots k_{s^{\prime}}^{\prime}\right\}=\left(\{1, \ldots m+1\}-\left\{k_{1}, \ldots k_{s}\right\}\right) \cup\left\{k_{l_{1}}, \ldots k_{l_{r}}\right\} .
$$

Then

$$
\begin{aligned}
& \left\{k_{1}^{\prime}, \ldots k_{s^{\prime}}^{\prime}\right\}-\left\{k_{l_{1}}, \ldots k_{l_{r}}\right\}=\{1, \ldots m+1\}-\left\{k_{1}, \ldots k_{s}\right\} \\
& \left\{k_{1}, \ldots k_{s}\right\}-\left\{k_{l_{1}}, \ldots k_{l_{r}}\right\}=\{1, \ldots m+1\}-\left\{k_{1}^{\prime}, \ldots k_{s^{\prime}}^{\prime}\right\}
\end{aligned}
$$

and $s^{\prime}=m+1-s+r$. By construction $\left\{k_{l_{1}}, \ldots k_{l_{r}}\right\} \subset\left\{k_{1}^{\prime}, \ldots k_{s^{\prime}}^{\prime}\right\}$, so we can consider the term

$$
\begin{aligned}
& (-1)^{\ell\left(k_{1}^{\prime}, \ldots k_{s^{\prime}}^{\prime}, 1, \ldots \hat{k}_{1}^{\prime}, \ldots \hat{k}_{s^{\prime}}^{\prime}, \ldots m+1\right)}(-1)^{\ell\left(k_{l_{1}} \ldots k_{l_{r}} k_{1}^{\prime} \ldots \hat{k}_{l_{1}} \ldots \hat{k}_{l_{r}} \ldots k_{s^{\prime}}^{\prime}\right)}
\end{aligned}
$$

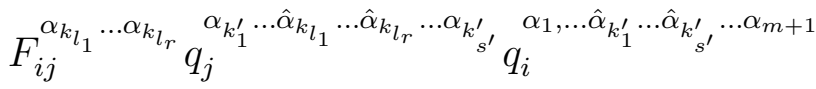

(21) is equal to (19) with $q_{i}$ in the place of $q_{j}$, except possibly by a sign. We are going to see that the sign is the same. We start by observing that

$$
\begin{aligned}
& \ell\left(k_{1} \ldots k_{s}, 1 \ldots \hat{k}_{1} \ldots \hat{k}_{s} \ldots m+1\right)=\ell\left(k_{l_{1}} \ldots k_{l_{r}}, 1 \ldots \hat{k}_{1} \ldots \hat{k}_{s} \ldots m+1\right)+ \\
& \ell\left(k_{1} \ldots \hat{k}_{l_{1}} \ldots \hat{k}_{l_{r}} \ldots k_{s}, 1 \ldots \hat{k}_{1} \ldots \hat{k}_{s} \ldots m+1\right), \\
& \ell\left(k_{1}^{\prime} \ldots k_{s^{\prime}}^{\prime}, 1 \ldots \hat{k}_{1}^{\prime} \ldots \hat{k}_{s^{\prime}}^{\prime} \ldots m+1\right)=\ell\left(k_{l_{1}} \ldots k_{l_{r}}, 1 \ldots \hat{k}_{1}^{\prime} \ldots \hat{k}_{s^{\prime}}^{\prime} \ldots m+1\right)+ \\
& \ell\left(k_{1}^{\prime} \ldots \hat{k}_{l_{1}} \ldots \hat{k}_{l_{r}} \ldots k_{s^{\prime}}^{\prime}, 1 \ldots \hat{k}_{1}^{\prime} \ldots \hat{k}_{s^{\prime}}^{\prime} \ldots m+1\right) .
\end{aligned}
$$

It is now crucial the fact that $s-r, m+1-s, s^{\prime}-r, m+1-s^{\prime}$ are all even numbers. The last two terms in the above equalities are easily seen equal modulo 2 by using (20). Then we have

$$
\begin{aligned}
& \ell\left(k_{1} \ldots k_{s}, 1 \ldots \hat{k}_{1} \ldots \hat{k}_{s} \ldots m+1\right)-\ell\left(k_{1}^{\prime} \ldots k_{s^{\prime}}^{\prime}, 1 \ldots \hat{k}_{1}^{\prime} \ldots \hat{k}_{s^{\prime}}^{\prime} \ldots m+1\right)= \\
& \ell\left(k_{l_{1}} \ldots k_{l_{r}}, 1 \ldots \hat{k}_{1} \ldots \hat{k}_{s} \ldots m+1\right)-\ell\left(k_{l_{1}} \ldots k_{l_{r}}, 1 \ldots \hat{k}_{1}^{\prime} \ldots \hat{k}_{s^{\prime}}^{\prime} \ldots m+1\right) \bmod 2= \\
& \ell\left(k_{l_{1}} \ldots k_{l_{r}}, k_{1}^{\prime}, \ldots \hat{k}_{l_{1}} \ldots \hat{k}_{l_{r}}, \ldots k_{s^{\prime}}^{\prime}\right)-\ell\left(k_{l_{1}} \ldots k_{l_{r}}, k_{1}, \ldots \hat{k}_{l_{1}} \ldots \hat{k}_{l_{r}}, \ldots k_{s}\right) \bmod 2,
\end{aligned}
$$


where we have used (20) again. We have proven our claim and all the terms in (18) that do not contain $q_{i}^{0}$ cancel. Then from (18) we deduce

$$
\begin{aligned}
& \left(f_{i}^{\alpha_{1} \ldots \alpha_{m+1}}-\sum_{s=0}^{m} \sum_{1 \leq k_{1}<\cdots<k_{s} \leq m+1}(-1)^{\ell\left(k_{1}, \ldots k_{s}, 1, \ldots \hat{k}_{1}, \ldots \hat{k}_{s}, \ldots m+1\right)}\right. \\
& \left.F_{i j}^{\alpha_{k_{1} \ldots \alpha_{k_{s}}}} q_{j}^{\alpha_{1}, \ldots \hat{\alpha}_{k_{1}} \ldots \hat{\alpha}_{k_{s}} \ldots \alpha_{m+1}}\right) q_{i}^{\circ}=0 .
\end{aligned}
$$

From the hypothesis of the theorem, this means that there exists $F_{i j}^{\alpha_{1} \ldots \alpha_{m+1}}$, antisymmetric in $i$ and $j$ such that

$$
\begin{aligned}
& f_{i}^{\alpha_{1} \ldots \alpha_{m+1}}-\sum_{s=0}^{m} \sum_{\substack{1 \leq k_{1}<\cdots<k_{s} \leq m+1 \\
F_{i j}}}(-1)^{\ell\left(k_{1}, \ldots k_{s}, 1, \ldots \hat{k}_{1}, \ldots \hat{k}_{s}, \ldots m+1\right)} \\
& F_{i}^{\alpha_{k_{1}} \ldots \alpha_{k_{s}}, \ldots \hat{\alpha}_{k_{1}} \ldots \hat{\alpha}_{k_{s}} \ldots \alpha_{m+1}}=F_{i j}^{\alpha_{1} \ldots \alpha_{m+1}} q_{j}^{\circ},
\end{aligned}
$$

from which one can compute

$f_{i}^{\alpha_{1} \ldots \alpha_{m+1}}=\sum_{s=0}^{m+1} \sum_{1 \leq k_{1}<\cdots<k_{s} \leq m+1}(-1)^{\ell\left(k_{1}, \ldots k_{s}, 1, \ldots \hat{k}_{1}, \ldots \hat{k}_{s}, \ldots m+1\right)} F_{i j}^{\alpha_{k_{1}} \ldots \alpha_{k_{s}}} q_{j}^{\alpha_{1}, \ldots \hat{\alpha}_{k_{1}} \ldots \hat{\alpha}_{k_{s}} \ldots \alpha_{m+1}}$

terminating our induction.

\section{Acknowledgments}

We would like to thank Prof. V. S. Varadarajan and Prof. A. Vistoli for many helpful comments.

Work supported in part by the European Community's Human Potential Program under contract HPRN-CT-2000-00131 Quantum Space-Time, M. A. Ll. is associated to Torino University.

The work of M. A Ll. has also been supported by the research grant BFM 2002-03681 from the Ministerio de Ciencia y Tecnología (Spain) and from EU FEDER funds.

\section{References}

[1] Y. A. Gol'fand and E. P. Likhtman, Extension of the Poincaré group and violation of $P$ invariance, JETP Lett. 13 (1971) 323. 
[2] J. Wess and B. Zumino, A Lagrangian model invariant under supergauge transformations Phys. Lett. B 49 (1974) 52.

[3] A. Salam and J. Strathdee, Nucl. Phys. B 76 (1974) 477-482.

[4] S. Ferrara, J. Wess and B. Zumino, Supergauge multiplets and superfields. Phys. Lett. B 51n. 3 (1974) 41.

[5] J. Wess and B. Zumino, Supergauge invariant extension of quantum electodynamics. Nucl. Phys. B 78 (1974) 1-13.

[6] S. Ferrara and B. Zumino, Supergauge invariant Yang-Mills theories. Nucl. Phys. B 79 (1974) 413-421.

[7] A. Salam and J. Strathdee, Phys. Lett. B 51 (1974) 353.

[8] S. Ferrara, D. Freedman and P. van Nieuwenhuizen, Progress towards a theory of supergravity. Phys. Rev. D 13 (1976) 3214-3218.

[9] S. Deser and B. Zumino, Consistent supergravity. Phys. Lett. B 62 (1976) 335.

[10] S. Ferrara, Supersymmetry and supergravity Reprint volume, World Scientific (1988).

[11] F. A. Berezin, Introduction to superanalysis. Edited by A. A. Kirillov. D. Reidel Publishing Company, Dordrecht (Holland) (1987). With an appendix by V. I. Ogievetsky. Translated from the Russian by J. Niederle and R. Kotecký. Translation edited by Dimitri Leĭtes.

[12] B. Kostant, Graded manifolds, Graded Lie theory and prequantization. Lecture Notes in Math. 570 (1977).

[13] D. A. Leites, Introduction to the theory of supermanifolds. Russian Math. Survey. 35:1 (1980) 1-64.

[14] Y. Manin, Gauge field theory and complex geometry. Springer Verlag, (1988).

[15] P. Deligne and J. Morgan, Notes on supersymmetry (following J. Bernstein), in "Quantum fields and strings. A course for mathematicians", Vol 1, AMS, (1999). 
[16] V. G. Kac, Lie superalgebras Adv. in Math. 26 (1977) 8-26.

[17] V. Rittenberg, A guide to Lie superalgebras. Invited Talk at Int. Colloquium on Group Theoretical Methods in Physics, Tubingen, Germany, Jul 18-22, (1977).

[18] D. Vogan, The Orbit Method and Unitary Representations for Reductive Lie groups, in "Algebraic and Analytic Methods in Representation Theory". Perspectives in Mathematics, Vol 17. Academic Press. (1996).

[19] J. C. Várilly and J. M. Gracia-Bondía, The Moyal representation for spin. Ann. of Phys. 190 (1989) 107-148.

[20] J. Madore, The fuzzy sphere. Class. Quantum Grav. 9 (1992) 69-87.

[21] S. Doplicher, K. Fredenhagen and J.E. Roberts The quantum structure of space-time at the Planck scale and quantum fields. Commun.Math.Phys. 172 (1995) 187-220.

[22] A. Connes, Non Commutative Geometry. Academic press. (1994).

[23] A. Connes, M. R. Douglas and A. Schwarz, Non commutative geometry and Matrix theory:Compactification on tori. JHEP 9802 (1998) 003.

[24] F. Bayen, M. Flato, C. Fronsdal, A. Lichnerowicz, D. Sternheimer, Deformation theory and quantization. I and II. Ann. of Phys. 111 1-151 (1978).

[25] B. Cahen, Deformation program for principal series representations. Lett. Math. Phys., 36, 65-75, (1996).

[26] D. Arnal, M. Cahen and S. Gutt, Deformations on coadjoint orbits. J. Geom. Phys. 3 (1986), 327-351.

[27] M. Cahen, S. Gutt, Produits * sur les orbites des groupes semi-simples de rang 1, C.R. Acad. Sc. Paris 296 (1983), série I, 821-823; An algebraic construction of $*$ product on the regular orbits of semisimple Lie groups. In Gravitation and Cosmology. Monographs and Textbooks in Physical Sciences. A volume in honor of Ivor Robinson, Bibliopolis. Eds W. Rundler and A. Trautman, (1987); Non localité d'une déformation symplectique sur la sphère $S^{2}$. Bull. Soc. Math. Belg. 36 B, 207-221, (1987). 
[28] R. Fioresi and M. A. Lledó, On the deformation Quantization of Coadjoint Orbits of Semisimple Lie Groups. Pacific J. of Math 198 No 2 411-436 (2001);

[29] R. Fioresi and M. A. Lledó, A Comparison between Star Products on Regular Orbits of Compact Lie Groups. To appear in the Journal of Physics A: Mathematical and General. math.QA/0106129.

R. Fioresi, A. Levrero and M. A. Lledó, Algebraic and Differential Star Products on Regular Orbits of Compact Lie Groups. To appear in the Pacific J. of Math. math.QA/0011172.

M. A. Lledó.Deformation Quantization of Non Regular Orbits of Compact Lie Groups. Lett. Math. Phys. 58 57-62 (2001).

[30] C. Fronsdal Some ideas about Quantization. Reports on Math. Phys. 15, 111-145 (1978).

[31] C. Moreno, Invariant star products and representations of compact semisimple Lie groups. Lett. Math. Phys. 12, 217-229 (1986).

[32] N. Seiberg and E. Witten, String Theory and Noncommutative Geometry. JHEP 09032 (1999).

[33] C. Klimcik, An extended fuzzy supersphere and twisted chiral superfields, Commun. Math. Phys. 206587 (1999)

[34] H. Grosse and G. Reiter, The fuzzy (super)sphere and field theory. Prepared for Conference on New Insights in Quantum Mechanics, Goslar, Germany, 31 Aug - 3 Sep 1998.

[35] A. P. Balachandran, S. Kurkcuoglu and E.Rojas, The star product on the fuzzy supersphere. JHEP 0207056 (2002).

[36] D. A. Leites, Spectra of graded commutative rings. Uspehi Matem. Nauk, 30 n. 3, 209 (1974). (In Russian).

[37] R. Hartshorne, Algebraic geometry. Springer Verlag, New York (USA) (1977).

[38] J. S. Milne, Algebraic geometry. http://www.jmilne.org/(1996). 
[39] I. R. Shafarevich, Basic algebraic geometry. Springer-Verlag Berlin Heidelberg (1977).

[40] R. Fioresi, On algebraic supergroups and quantum deformations, math.QA/0111113.

Supergroups, quantum supergroups and their homogeneous spaces. Euroconference on Brane New World and Noncommutative Geometry (Torino, 2000). Modern Phys. Lett. A 16 269-274 (2001).

[41] D. Eisenbud and J. Harris, The geometry of schemes. Springer Verlag, New York (2000).

[42] M. Demazure and P. Gabriel, Groupes Algébriques, Tome 1. Mason\&Cie, éditeur. North-Holland Publishing Company, The Netherlands (1970). (Page 341).ATENTION!!

[43] A. Borel, Linear algebraic groups. Springer Verlag, New York (1991).

[44] V. G. Kac, Characters of typical representations of classical Lie superalgebras. Commun. Alg. 5 (1977) 889-897;

A. Sergeev, The invariant polynomials on simple Lie superalgebras, represent. Theory 3 (1999) 250-280;

D. Leites and A. Sergeev, Casimir operators for Lie superalgebras. arXiv:math.RT/0202180.

[45] P. J. Olver, Applications of Lie groups to differential equations, Springer verlag (1986).

[46] V. S. Varadarajan, On the ring of invariant polynomials on a semisimple Lie algebra. Amer. J. Math, 90, (1968).

[47] Y. Manin, Topics in non commutative geometry. Princeton University Press, (1991). 